\title{
Quadruple random common fixed point results of general- ized Lipschitz mappings in cone b-metric spaces over Banach algebras
}

\begin{abstract}
Chayut Kongbana,c, Poom Kumamª,b,c,*
${ }^{a}$ KMUTTFixed Point Research Laboratory, Department of Mathematics, Room SCL 802 Fixed Point Laboratory, Science Laboratory Building, Faculty of Science, King Mongkuts University of Technology Thonburi (KMUTT), 126 Pracha-Uthit Road, Bang Mod, Thrung Khru, Bangkok 10140, Thailand.

${ }^{b}$ Department of Medical Research, China Medical University Hospital, China Medical University, Taichung 40402, Taiwan.

${ }^{c}$ KMUTT-Fixed Point Theory and Applications Research Group (KMUTT-FPTA), Theoretical and Computational Science Center (TaCS), Science Laboratory Building, Faculty of Science, King Mongkuts University of Technology Thonburi (KMUTT), 126 Pracha-Uthit Road, Bang Mod, Thrung Khru, Bangkok 10140, Thailand.
\end{abstract}

Communicated by Y. H. Yao

\begin{abstract}
In this paper, we introduce the concept of cone b-metric spaces over Banach algebras and present some quadruple random coincidence points and quadruple random common fixed point theorems for nonlinear operators in such spaces.
\end{abstract}

Keywords: Quadruple random fixed point, quadruple common random fixed point, quadruple random coincidence point, cone b-metric space over Banach algebra.

2010 MSC: 47H10, 60H25.

(C)2018 All rights reserved.

\section{Introduction}

It is well-known that the classic contraction mapping principle of Banach is a fundamental result in fixed point theory. Several authors have obtained various extensions and generalizations of Banach's theorem by considering contractive mappings on many different metric spaces. For example, [3, 5, 23, 37], and others. In 1989, Bakhtin [4] introduced b-metric space as a generalization of usual metric space and proved the contraction mapping principle in b-metric space. In 2007, Huang and Zhang [10] introduced the concept of cone metric space as generalization of metric and proved some fixed theorems of contractive mapping on complete cone metric space with the assumption of normality of a cone. Fixed point theorems for contractive mapping on b-metric space and cone metric have been proved by several authors (see

\footnotetext{
*Corresponding author

Email addresses: chayut_@hotmail.com (Chayut Kongban), poom.kum@kmutt.ac.th (Poom Kumam)

doi: 10.22436/jnsa.011.01.10
}

Received: 2017-05-31 Revised: 2017-09-14 Accepted: 2017-11-06 
$[2,7,14,25-27,29,30,32-34])$. In 2011, Hussain and Shah [12] introduced the concept of cone b-metric space as a generalization of cone metric space and b-metric space. In 2013, Liu and Xu [21] introduced cone metric space over Banach algebra by replacing Banach spaces with Banach algebra and proved some fixed point theorems of generalized Lipschitz mapping with weaker conditions on generalized Lipschitz constants. In 2015, Huang and Radenović [9] introduced the concept of cone b-metric space over Banach algebra and proved some common fixed point theorems of generalized Lipschitz mappings in such setting without assumption of normality. Random fixed point theorems are stochastic generalizations of classical fixed point theorems. On the other hand, in 2009, Ćirić and Lakshmikantham [6] proved some coupled random coincidence and coupled random fixed points in partially ordered metric space. Afterwards, many authors have focused on random fixed point theorems in complete separable metric space and coupled random coincidence and coupled random fixed points in partially ordered metric space. A large number of works are noted in $[1,11,16-20,31,35,36]$ and the relevant literature therein. Recently, Jiang et al. [13] proved tripled random coincidence point and common fixed point results of generalized Lipschitz mappings in cone b-metric spaces over Banach algebras. In this paper, we present several quadruple random common fixed points theorems with several generalized Lipschitz constants of cone b-metric spaces over Banach algebras.

\section{Preliminaries}

Let $\mathcal{A}$ be a Banach algebra with a unit $e$, and $\theta$ the zero element of $\mathcal{A}$. A nonempty closed convex subset $\mathrm{P}$ of $\mathcal{A}$ is called a cone if

1. $\{\theta, e\} \subset P$;

2. $\mathrm{P}^{2}=\mathrm{PP} \subset \mathrm{P}, \mathrm{P} \cap(-\mathrm{P})=\{\theta\}$;

3. $\lambda \mathrm{P}+\mu \mathrm{P} \subset \mathrm{P}$ for all $\lambda, \mu \geqslant 0$.

On this basis, we define a partial ordering $\preceq$ with respect to $P$ by $x \preceq y$ if and only if $y-x \in P$. We shall write $x \prec y$ to indicate that $x \preceq y$ but $x \neq y$, while $x \ll y$ will indicate that $y-x \in \operatorname{int} P$, where int $(P)$ stands for the interior of $\mathrm{P}$. Write $\|\cdot\|$ as the norm on $\mathcal{A}$. A cone $\mathrm{P}$ is called normal if there is a number $M>0$ such that for all $x, y \in \mathcal{A}$,

$$
\theta \preceq x \preceq y \Longrightarrow\|x\| \leqslant M\|y\| .
$$

The least positive number satisfying above is called the normal constant of $\mathrm{P}$.

In the following we always suppose that $\mathcal{A}$ is a Banach algebra with a unit e. $\mathrm{P}$ is a cone in $\mathcal{A}$ with $\operatorname{int}(\mathrm{P}) \neq \emptyset$, and $\preceq$ is a partial ordering with respect to $\mathrm{P}$.

Definition 2.1. Let $\mathrm{X}$ be a nonempty set and $\mathcal{A}$ a Banach algebra. Suppose that the mapping $\mathrm{d}: \mathrm{X} \times \mathrm{X} \rightarrow \mathcal{A}$ satisfies:

1. $\theta \prec d(x, y)$ for all $x, y \in X$ with $x \neq y$ and $d(x, y)=\theta$ if and only if $x=y$;

2. $d(x, y)=d(y, x)$ for all $x, y \in X$;

3. $d(x, y) \preceq s(d(x, z)+d(z, y))$ for all $x, y, z \in X$,

where $s \geqslant 1$ is a constant. Then $d$ is called a cone $b$-metric on $X$, and $(X, d)$ is called a cone $b$-metric space over Banach algebra $\mathcal{A}$.

Definition $2.2([12])$. Let $(X, d)$ be a cone b-metric space over Banach algebra, $x \in X$ and $\left\{x_{n}\right\}$ a sequence in X. Then

1. $\left\{x_{n}\right\}$ converges to $x$ whenever, for every $c \in E$ with $\theta \ll c$ there is a natural number $N$ such that $d\left(x_{n}, x\right) \ll c$ for all $n>N$. We denote this by $\lim _{n \rightarrow \infty} x_{n}=x$ or $x_{n} \rightarrow x(n \rightarrow \infty)$.

2. $\left\{x_{n}\right\}$ is a Cauchy sequence whenever, for every $c \in E$ with $\theta \ll c$ there is a natural number $N$ such that $d\left(x_{n}, x_{m}\right) \ll c$ for all $n, m \geqslant N$.

3. $(X, d)$ is complete if every Cauchy sequence is convergent.

The following lemmas are often used (in particular when dealing with cone b-metric spaces in which the cones need not to be normal). 
Lemma 2.3 ([12]). Let $(\mathrm{X}, \mathrm{d})$ be a cone b-metric space over Banach algebra $\mathcal{A}$ and $\mathrm{P}$ a cone in $\mathcal{A}$. Then the following properties are often used.

(1) If $c \in \operatorname{int}(P)$ and $\theta \preceq a_{n} \rightarrow \theta(n \rightarrow \infty)$, then there exists $N$ such that for all $n>N$, we have $a_{n} \ll c$.

(2) If $x \preceq y$ and $y \ll z$, then $x \ll z$.

(3) If $\theta \preceq u \ll c$ for each $c \in \operatorname{int}(P)$, then $u=\theta$.

(4) If $\mathrm{u} \in \mathrm{P}$ and $\mathrm{u} \preceq \mathrm{ku}$ for some $0 \leqslant \mathrm{k}<1$, then $\mathrm{u}=\theta$.

(5) If $\mathrm{a} \preceq \mathrm{b}+\mathrm{c}$ for each $\mathrm{c} \in \operatorname{int}(\mathrm{P})$, then $\mathrm{a} \preceq \mathrm{b}$.

(6) Let $\theta \ll c$. If $\theta \preceq d\left(x_{n}, x\right) \preceq b_{n}$ and $b_{n} \rightarrow \theta(n \rightarrow \infty)$, then $d\left(x_{n}, x\right) \ll c$, where $\left\{x_{n}\right\}, x$ are a sequence and a given point in $X$, respectively.

Lemma 2.4 ([28]). Let $\mathcal{A}$ be a Banach algebra with a unit e and $x \in \mathcal{A}$, then $\lim _{n \rightarrow \infty}\left\|x^{\mathfrak{n}}\right\|^{\frac{1}{n}}$ exists and the spectral radius $\rho(x)$ satisfies

$$
\rho(x)=\lim _{n \rightarrow \infty}\left\|x^{\mathfrak{n}}\right\|^{\frac{1}{n}}=\inf \left\|x^{\mathfrak{n}}\right\|^{\frac{1}{n}} .
$$

If $\rho(x)<|\lambda|$, then $\lambda e-x$ is invertible in $\mathcal{A}$, moreover,

$$
(\lambda e-x)^{-1}=\sum_{i=0}^{\infty} \frac{x^{i}}{\lambda^{i+1}},
$$

where $\lambda$ is a complex constant.

Lemma 2.5 ([28]). Let $\mathcal{A}$ be a Banach algebra with a unit $\mathrm{e}$, and $\mathrm{a}, \mathrm{b} \in \mathcal{A}$. If $\mathrm{a}$ commutes with $\mathrm{b}$, then

$$
\rho(a+b) \leqslant \rho(a)+\rho(b), \rho(a b) \leqslant \rho(a) \rho(b) .
$$

Lemma 2.6 ([38]). Let $\mathrm{P}$ be a cone in a Banach algebra $\mathcal{A}$ and $\mathrm{k} \in \mathrm{P}$ be a given vector. Let $\left\{\mathrm{u}_{\mathrm{n}}\right\}$ be a sequence in P. If for each $c_{1} \gg \theta$, there exists $\mathrm{N}_{1}$ such that $\mathrm{u}_{\mathrm{n}} \ll \mathrm{c}_{1}$ for all $\mathrm{n}>\mathrm{N}_{1}$, then for each $\mathrm{c}_{2} \gg \theta$, there exists $\mathrm{N}_{2}$ such that $\mathrm{ku} \mathrm{u}_{\mathrm{n}} \ll \mathrm{c}_{2}$ for all $\mathrm{n}>\mathrm{N}_{2}$.

Lemma 2.7 ([38]). Let $\mathcal{A}$ be a Banach algebra and $\mathrm{k} \in \mathcal{A}$. If $\rho(\mathrm{k})<1$ then $\lim _{\mathrm{n} \rightarrow \infty}\left\|\mathrm{k}^{\mathrm{n}}\right\|=0$.

Lemma 2.8 ([38]). Let $\mathcal{A}$ be a Banach algebra with a unit e, and $\left\{x_{n}\right\}$ a sequence in $\mathcal{A}$. If $\left\{x_{n}\right\}$ converges to $x$ in $\mathcal{A}$, and for any $n \geqslant 1,\left\{x_{n}\right\}$ commutes with $x$, then $\rho\left(x_{n}\right) \rightarrow \rho(x)$ as $n \rightarrow \infty$

Definition 2.9 ([15]). An element $(x, y, z, w) \in X^{4}$ is said to be a quadruple fixed point of the mapping $\mathrm{F}: \mathrm{X}^{4} \rightarrow \mathrm{X}$ if $\mathrm{F}(\mathrm{x}, \mathrm{y}, z, w)=x, \mathrm{~F}(\mathrm{y}, z, w, x)=y, \mathrm{~F}(z, w, x, y)=z$, and $\mathrm{F}(w, x, y, z)=w$.

Definition 2.10 ([24]). Let $X$ be a nonempty set and let $F: X^{4} \rightarrow X, g: X \rightarrow X$. An element $(x, y, z, w) \in X$ is called

(1) a quadruple coincidence point of $F$ and $g$ if $F(x, y, z, w)=g x, F(y, z, w, x)=g y, F(z, w, x, y)=g z$, and $F(w, x, y, z)=g w ;(g x, g y, g z, g w)$ is said to be a quadruple point of coincidence of $F$ and $g$.

(2) a quadruple common fixed point of $F$ and $g$ if $F(x, y, z, w)=g x=x, F(y, z, w, x)=g y=y$, $\mathrm{F}(z, w, x, y)=g z=z$, and $F(w, x, y, z)=g w=w$.

Definition 2.11. The mappings $F: X^{4} \rightarrow X$ and $g: X \rightarrow X$ are called $w$-compatible if $g(F(x, y, z, w))=$ $\mathrm{F}(\mathrm{gx}, \mathrm{gy}, \mathrm{gz}, \mathrm{gw})$ whenever $\mathrm{gx}=\mathrm{F}(\mathrm{x}, y, z, w), g y=\mathrm{F}(y, z, w, x), \mathrm{g} z=\mathrm{F}(z, w, x, y)$, and $g w=F(w, x, y, z)$.

Let $(\Omega, \Sigma)$ be a measurable space with $\Sigma$ a sigma algebra of subsets of $\Omega$ and let $(X, d)$ be a metric space. A mapping $T: \Omega \rightarrow X$ is called $\Sigma$-measurable if for any open subset $U$ of $X, T^{-1}(U)=\{\omega: T(\omega) \in U\} \in \Sigma$ (see [8]). In what follows, when we speak of measurability we shall mean $\Sigma$-measurability. A mapping $\mathrm{T}: \Omega \times \mathrm{X} \rightarrow \mathrm{X}$ is called a random operator if for any $\mathrm{x} \in \mathrm{X}, \mathrm{T}(\cdot, x)$ is measurable. A measurable mapping $\xi: \Omega \rightarrow X$ is called a random fixed point of a random operator $T: \Omega \times X \rightarrow X$, if $\xi(\omega)=T(\omega, \xi(\omega))$ for every $\omega \in \Omega$.

Theorem 2.12 ([8]). Let $\mathrm{X}$ be separable metric, $\mathrm{Y}$ be metric, $\mathrm{f}: \Omega \times \mathrm{X} \rightarrow \mathrm{Y}$ a function measurable in $\omega$ and continuous in $\mathrm{X}, \Gamma: \Omega \rightarrow \mathrm{X}$ a measurable multifunction with compact values, and $\mathrm{g}: \Omega \rightarrow \mathrm{Y}$ a measurable function such that $\mathrm{g}(\omega) \in \mathrm{f}(\{\omega\} \times \Gamma(\omega))$ for all $\omega \in \Omega$. Then there exists a measurable selector $\gamma: \Omega \rightarrow X$ for $\Gamma$ such that $\mathrm{g}(\omega)=\mathrm{f}(\omega, \gamma(\omega))$ for all $\omega \in \Omega$. 
Definition 2.13. Let $(X, d)$ be a separable metric space and $(\Omega, \Sigma)$ be a measurable space. Then $F: X^{4} \rightarrow X$ and $g: X \rightarrow X$ are said to be $w$-compatible random operators if

$$
F(\omega,(g(\omega, x), g(\omega, y), g(\omega, z), g(\omega, w)))=g(\omega, F(\omega,(x, y, z, w))),
$$

whenever $F(\omega,(x, y, z, w))=g(\omega, x), F(\omega,(y, z, w, x))=g(\omega, y), F(\omega,(z, w, x, y))=g(\omega, z), F(\omega,(w, x, y, z))$ $=g(\omega, w)$ for all $\omega \in \Omega$ and $x, y, z, w \in X$ are satisfied.

\section{Main results}

Theorem 3.1. Let $(\mathrm{X}, \mathrm{d})$ be a separable cone b-metric space over Banach algebra $\mathcal{A}$ and $\mathrm{P}$ be a cone in $\mathcal{A}, \mathrm{s} \geqslant 1$ be a constant, and $(\Omega, \Sigma)$ be a measurable space. Suppose that the mappings $F: \Omega \times X^{4} \rightarrow X, g: \Omega \times X \rightarrow X$ satisfy the following contractive condition:

$$
\begin{aligned}
d(F(\omega) & \left.\left.\left(x_{1}, x_{2}, x_{3}, x_{4}\right)\right), F\left(\omega,\left(x_{1}^{*}, x_{2}^{*}, x_{3}^{*}, x_{4}^{*}\right)\right)\right) \\
\preceq & {\left[a_{1} d\left(g\left(\omega, x_{1}\right), F\left(\omega,\left(x_{1}, x_{2}, x_{3}, x_{4}\right)\right)\right)+a_{2} d\left(g\left(\omega, x_{2}\right), F\left(\omega,\left(x_{2}, x_{3}, x_{4}, x_{1}\right)\right)\right)\right.} \\
& \left.+a_{3} d\left(g\left(\omega, x_{3}\right), F\left(\omega,\left(x_{3}, x_{4}, x_{1}, x_{2}\right)\right)\right)+a_{4} d\left(g\left(\omega, x_{4}\right), F\left(\omega,\left(x_{4}, x_{1}, x_{2}, x_{3}\right)\right)\right)\right] \\
& +\left[a_{5} d\left(g\left(\omega, x_{1}^{*}\right), F\left(\omega,\left(x_{1}^{*}, x_{2}^{*}, x_{3}^{*}, x_{4}^{*}\right)\right)\right)+a_{6} d\left(g\left(\omega, x_{2}^{*}\right), F\left(\omega,\left(x_{2}^{*}, x_{3}^{*}, x_{4}^{*}, x_{1}^{*}\right)\right)\right)\right. \\
& \left.+a_{7} d\left(g\left(\omega, x_{3}^{*}\right), F\left(\omega,\left(x_{3}^{*}, x_{4}^{*}, x_{1}^{*}, x_{2}^{*}\right)\right)\right)+a_{8} d\left(g\left(\omega, x_{4}^{*}\right), F\left(\omega,\left(x_{4}^{*}, x_{1}^{*}, x_{2}^{*}, x_{3}^{*}\right)\right)\right)\right] \\
& +\left[a_{9} d\left(g\left(\omega, x_{1}\right), F\left(\omega,\left(x_{1}^{*}, x_{2}^{*}, x_{3}^{*}, x_{4}^{*}\right)\right)\right)+a_{10} d\left(g\left(\omega, x_{2}\right), F\left(\omega,\left(x_{2}^{*}, x_{3}^{*}, x_{4}^{*}, x_{1}^{*}\right)\right)\right)\right. \\
& \left.+a_{11} d\left(g\left(\omega, x_{3}\right), F\left(\omega,\left(x_{3}^{*}, x_{4}^{*}, x_{1}^{*}, x_{2}^{*}\right)\right)\right)+a_{12} d\left(g\left(\omega, x_{4}\right), F\left(\omega,\left(x_{4}^{*}, x_{1}^{*}, x_{2}^{*}, x_{3}^{*}\right)\right)\right)\right] \\
& +\left[a_{13} d\left(g\left(\omega, x_{1}^{*}\right), F\left(\omega,\left(x_{1}, x_{2}, x_{3}, x_{4}\right)\right)\right)+a_{14} d\left(g\left(\omega, x_{2}^{*}\right), F\left(\omega,\left(x_{2}, x_{3}, x_{4}, x_{1}\right)\right)\right)\right. \\
& \left.+a_{15} d\left(g\left(\omega, x_{3}^{*}\right), F\left(\omega,\left(x_{3}, x_{4}, x_{1}, x_{2}\right)\right)\right)+a_{16} d\left(g\left(\omega, x_{4}^{*}\right), F\left(\omega,\left(x_{4}, x_{1}, x_{2}, x_{3}\right)\right)\right)\right] \\
& +a_{17} d\left(g\left(\omega, x_{1}\right), g\left(\omega, x_{1}^{*}\right)\right)+a_{18} d\left(g\left(\omega, x_{2}\right), g\left(\omega, x_{2}^{*}\right)\right) \\
& +a_{19} d\left(g\left(\omega, x_{3}\right), g\left(\omega, x_{3}^{*}\right)\right)+a_{20} d\left(g\left(\omega, x_{4}\right), g\left(\omega, x_{4}^{*}\right)\right)
\end{aligned}
$$

for all $x_{1}, x_{2}, x_{3}, x_{4}, x_{1}^{*}, x_{2}^{*}, x_{3}^{*}, x_{4}^{*}$, where $a_{i} \in P, a_{i} a_{j}=a_{j} a_{i}(i, j=1, \ldots, 20)$ are generalized Lipschitz constants with $(s+1) \rho\left(a_{1}+\cdots+a_{8}\right)+s(s+1) \rho\left(a_{9}+\cdots+a_{16}\right)+2 s \rho\left(a_{17}+\cdots+a_{20}\right)<2$ and $\rho\left(s a_{1}+s a_{2}+s a_{3}+\right.$ $\left.s a_{4}+s^{2} a_{13}+s^{2} a_{14}+s^{2} a_{15}+s^{2} a_{16}\right)<1$, where $s \geqslant 1$ is a constant. Let $F(\cdot, v), g(\cdot, x)$ are measurable for $v \in x^{4}$ and $x \in X$, respectively, $\mathrm{F}\left(\omega \times \mathrm{X}^{4}\right) \subseteq \mathrm{g}(\omega \times \mathrm{X})$ is complete subspace of $\mathrm{X}$ for each $\omega \in \Omega$, then there are mappings $\gamma, \zeta, \xi, \rho: \Omega \rightarrow X$, such that

$$
\begin{array}{ll}
F(\omega,(\gamma(\omega), \zeta(\omega), \xi(\omega), \rho(\omega)))=g(\omega, \gamma(\omega)), & F(\omega,(\zeta(\omega), \xi(\omega), \rho(\omega), \gamma(\omega)))=g(\omega, \zeta(\omega)), \\
F(\omega,(\xi(\omega), \rho(\omega), \gamma(\omega), \zeta(\omega)))=g(\omega, \xi(\omega)), & F(\omega,(\rho(\omega), \gamma(\omega), \zeta(\omega), \xi(\omega)))=g(\omega, \rho(\omega))
\end{array}
$$

for all $\omega \in \Omega$, that is $\mathrm{F}$ and $\mathrm{g}$ have a quadruple random coincidence point.

Proof. Let $\Theta=\{\eta: \Omega \rightarrow X\}$ be a family of measurable mappings. We construct four sequences of measurable mappings $\left\{\gamma_{n}\right\},\left\{\zeta_{n}\right\},\left\{\xi_{n}\right\},\left\{\rho_{n}\right\}$ in $\Theta$ and four sequences $\left\{g\left(\omega, \gamma_{n}(\omega)\right)\right\},\left\{g\left(\omega, \zeta_{n}(\omega)\right)\right\},\left\{g\left(\omega, \xi_{n}(\omega)\right)\right\}$, $\left\{g\left(\omega, \rho_{n}(\omega)\right)\right\}$ in $X$ as follows. Let $\gamma_{0}, \zeta_{0}, \xi_{0}, \rho_{0} \in \Theta$. Since $F\left(\omega,\left(\gamma_{0}(\omega), \zeta_{0}(\omega), \xi_{0}(\omega), \rho_{0}(\omega)\right)\right) \in F\left(\omega \times X^{4}\right) \subseteq$ $g(\omega, X)$, by a sort of Filippov measurable implicit function theorems (see [8,22]), there is $\gamma_{1} \in \Theta$ such that

$$
g\left(\omega, \gamma_{1}(\omega)\right)=F\left(\omega,\left(\gamma_{0}(\omega), \zeta_{0}(\omega), \xi_{0}(\omega), \rho_{0}(\omega)\right)\right) .
$$

Similarly as $F\left(\omega,\left(\zeta_{0}(\omega), \xi_{0}(\omega), \rho_{0}(\omega), \gamma_{0}(\omega)\right)\right) \subseteq g(\omega, X)$, there is $\zeta_{1} \in \Theta$ such that

$$
g\left(\omega, \zeta_{1}(\omega)\right)=F\left(\omega,\left(\zeta_{0}(\omega), \xi_{0}(\omega), \rho_{0}(\omega), \gamma_{0}(\omega)\right)\right) .
$$

Similarly as $F\left(\omega,\left(\xi_{0}(\omega), \rho_{0}(\omega), \gamma_{0}(\omega), \zeta_{0}(\omega)\right)\right) \subseteq g(\omega, X)$, there is $\xi_{1} \in \Theta$ such that

$$
g\left(\omega, \xi_{1}(\omega)\right)=F\left(\omega,\left(\xi_{0}(\omega), \rho_{0}(\omega), \gamma_{0}(\omega), \zeta_{0}(\omega)\right)\right) .
$$

Similarly as $F\left(\omega,\left(\rho_{0}(\omega), \gamma_{0}(\omega), \zeta_{0}(\omega), \xi_{0}(\omega)\right)\right) \subseteq g(\omega, X)$, there is $\rho_{1} \in \Theta$ such that

$$
g\left(\omega, \rho_{1}(\omega)\right)=F\left(\omega,\left(\rho_{0}(\omega), \gamma_{0}(\omega), \zeta_{0}(\omega), \xi_{0}(\omega)\right)\right) .
$$


Continuing this process, we obtain four sequences $\left\{\gamma_{n}(\omega)\right\},\left\{\zeta_{n}(\omega)\right\},\left\{\xi_{n}(\omega)\right\}$, and $\left\{\rho_{n}(\omega)\right\}$ in $X$ such that

$$
\begin{aligned}
& g\left(\omega,\left(\gamma_{n+1}(\omega)\right)\right)=F\left(\omega,\left(\gamma_{n}(\omega), \zeta_{n}(\omega), \xi_{n}(\omega), \rho_{n}(\omega)\right)\right), \\
& g\left(\omega,\left(\zeta_{n+1}(\omega)\right)\right)=F\left(\omega,\left(\zeta_{n}(\omega), \xi_{n}(\omega), \rho_{n}(\omega), \gamma_{n}(\omega)\right)\right), \\
& g\left(\omega,\left(\xi_{n+1}(\omega)\right)\right)=F\left(\omega,\left(\xi_{n}(\omega), \rho_{n}(\omega), \gamma_{n}(\omega), \zeta_{n}(\omega)\right)\right), \\
& g\left(\omega,\left(\rho_{n+1}(\omega)\right)\right)=F\left(\omega,\left(\rho_{n}(\omega), \gamma_{n}(\omega), \zeta_{n}(\omega), \xi_{n}(\omega)\right)\right)
\end{aligned}
$$

for all $n \in \mathbb{N}$. According to (3.1), we have

$$
\begin{aligned}
& \mathrm{d}\left(\mathrm{g}\left(\omega, \gamma_{\mathrm{n}}(\omega)\right), \mathrm{g}\left(\omega, \gamma_{\mathrm{n}+1}(\omega)\right)\right) \\
& =\mathrm{d}\left(\mathrm{F}\left(\omega,\left(\gamma_{n-1}(\omega), \zeta_{n-1}(\omega), \xi_{n-1}(\omega), \rho_{n-1}(\omega)\right)\right), \mathrm{F}\left(\omega,\left(\gamma_{n}(\omega), \zeta_{n}(\omega), \xi_{n}(\omega), \rho_{n}(\omega)\right)\right)\right) \\
& \preceq\left[a _ { 1 } d \left(g\left(\omega, \gamma_{n-1}(\omega)\right), F\left(\omega,\left(\gamma_{n-1}(\omega), \zeta_{n-1}(\omega), \xi_{n-1}(\omega), \rho_{n-1}(\omega)\right)\right)\right.\right. \\
& +a_{2} d\left(g\left(\omega, \zeta_{n-1}(\omega)\right), F\left(\omega,\left(\zeta_{n-1}(\omega), \xi_{n-1}(\omega), \rho_{n-1}(\omega), \gamma_{n-1}(\omega)\right)\right)\right. \\
& +a_{3} d\left(g\left(\omega, \xi_{n-1}(\omega)\right), F\left(\omega,\left(\xi_{n-1}(\omega), \rho_{n-1}(\omega), \gamma_{n-1}(\omega), \zeta_{n-1}(\omega)\right)\right)\right. \\
& +a_{4} d\left(g\left(\omega, \rho_{n-1}(\omega)\right), F\left(\omega,\left(\rho_{n-1}(\omega), \gamma_{n-1}(\omega), \zeta_{n-1}(\omega),\left(\xi_{n-1}(\omega)\right)\right)\right]\right. \\
& +\left[a _ { 5 } d \left(g\left(\omega, \gamma_{n}(\omega)\right), F\left(\omega,\left(\gamma_{n}(\omega), \zeta_{n}(\omega), \xi_{n}(\omega), \rho_{n}(\omega)\right)\right)\right.\right. \\
& +a_{6} d\left(g\left(\omega, \zeta_{n}(\omega)\right), F\left(\omega,\left(\zeta_{n}(\omega), \xi_{n}(\omega), \rho_{n}(\omega), \gamma_{n}(\omega)\right)\right)\right. \\
& +a_{7} d\left(g\left(\omega, \xi_{n}(\omega)\right), F\left(\omega,\left(\xi_{n}(\omega), \rho_{n}(\omega), \gamma_{n}(\omega), \zeta_{n}(\omega)\right)\right)\right. \\
& +a_{8} d\left(g\left(\omega, \rho_{n}(\omega)\right), F\left(\omega,\left(\rho_{n}(\omega), \gamma_{n}(\omega), \zeta_{n}(\omega),\left(\xi_{n}(\omega)\right)\right)\right]\right. \\
& +\left[a _ { 9 } d \left(g\left(\omega, \gamma_{n-1}(\omega)\right), F\left(\omega,\left(\gamma_{n}(\omega), \zeta_{n}(\omega), \xi_{n}(\omega), \rho_{n}(\omega)\right)\right)\right.\right. \\
& +a_{10} d\left(g\left(\omega, \zeta_{n-1}(\omega)\right), F\left(\omega,\left(\zeta_{n}(\omega), \xi_{n}(\omega), \rho_{n}(\omega), \gamma_{n}(\omega)\right)\right)\right. \\
& +a_{11} d\left(g\left(\omega, \xi_{n-1}(\omega)\right), F\left(\omega,\left(\xi_{n}(\omega), \rho_{n}(\omega), \gamma_{n}(\omega), \zeta_{n}(\omega)\right)\right)\right. \\
& +a_{12} d\left(g\left(\omega, \rho_{n-1}(\omega)\right), F\left(\omega,\left(\rho_{n}(\omega), \gamma_{n}(\omega), \zeta_{n}(\omega), \xi_{n}(\omega)\right)\right)\right] \\
& +\left[a _ { 1 3 } d \left(g\left(\omega, \gamma_{n}(\omega)\right), F\left(\omega,\left(\gamma_{n-1}(\omega), \zeta_{n-1}(\omega), \xi_{n-1}(\omega), \rho_{n-1}(\omega)\right)\right)\right.\right. \\
& +a_{14} d\left(g\left(\omega, \zeta_{n}(\omega)\right), F\left(\omega,\left(\zeta_{n-1}(\omega), \xi_{n-1}(\omega), \rho_{n-1}(\omega), \gamma_{n-1}(\omega)\right)\right)\right. \\
& +a_{15} d\left(g\left(\omega, \xi_{n}(\omega)\right), F\left(\omega,\left(\xi_{n-1}(\omega), \rho_{n-1}(\omega), \gamma_{n-1}(\omega), \zeta_{n-1}(\omega)\right)\right)\right. \\
& +a_{16} d\left(g\left(\omega, \rho_{n}(\omega)\right), F\left(\omega,\left(\rho_{n-1}(\omega), \gamma_{n-1}(\omega), \zeta_{n-1}(\omega), \xi_{n-1}(\omega)\right)\right)\right] \\
& +\left[a_{17} d\left(g\left(\omega, \gamma_{n-1}(\omega)\right), g\left(\omega, \gamma_{n}(\omega)\right)\right)+a_{18} d\left(g\left(\omega, \zeta_{n-1}(\omega)\right), g\left(\omega, \zeta_{n}(\omega)\right)\right)\right. \\
& \left.+a_{19} d\left(g\left(\omega, \xi_{n-1}(\omega)\right), g\left(\omega, \xi_{n}(\omega)\right)\right)+a_{20} d\left(g\left(\omega, \rho_{n-1}(\omega)\right), g\left(\omega, \rho_{n}(\omega)\right)\right)\right] \\
& \preceq\left[a_{1} d\left(g\left(\omega, \gamma_{n-1}(\omega)\right), g\left(\omega, \gamma_{n}(\omega)\right)\right)+a_{2} d\left(g\left(\omega, \zeta_{n-1}(\omega)\right), g\left(\omega, \zeta_{n}(\omega)\right)\right)\right. \\
& +a_{3} d\left(g\left(\omega, \xi_{n-1}(\omega)\right), g\left(\omega, \xi_{n}(\omega)\right)\right)+a_{4} d\left(g\left(\omega, \rho_{n-1}(\omega)\right), g\left(\omega, \rho_{n}(\omega)\right)\right) \\
& +\left[a_{5} d\left(g\left(\omega, \gamma_{n}(\omega)\right), g\left(\omega, \gamma_{n+1}(\omega)\right)\right)+a_{6} d\left(g\left(\omega, \zeta_{n}(\omega)\right), g\left(\omega, \zeta_{n+1}(\omega)\right)\right)\right. \\
& \left.+a_{7} d\left(g\left(\omega, \xi_{n}(\omega)\right), g\left(\omega, \xi_{n+1}(\omega)\right)\right)+a_{8} d\left(g\left(\omega, \rho_{n}(\omega)\right), g\left(\omega, \rho_{n+1}(\omega)\right)\right)\right] \\
& +\left[a_{9} d\left(g\left(\omega, \gamma_{n-1}(\omega)\right), g\left(\omega, \gamma_{n+1}(\omega)\right)\right)+a_{10} d\left(g\left(\omega, \zeta_{n-1}(\omega)\right), g\left(\omega, \zeta_{n+1}(\omega)\right)\right)\right. \\
& \left.+a_{11} d\left(g\left(\omega, \xi_{n-1}(\omega)\right), g\left(\omega, \xi_{n+1}(\omega)\right)\right)+a_{12} d\left(g\left(\omega, \rho_{n-1}(\omega)\right), g\left(\omega, \rho_{n+1}(\omega)\right)\right)\right] \\
& +\left[a_{13} d\left(g\left(\omega, \gamma_{n}(\omega)\right), g\left(\omega, \gamma_{n}(\omega)\right)\right)+a_{14} d\left(g\left(\omega, \zeta_{n}(\omega)\right), g\left(\omega, \zeta_{n}(\omega)\right)\right)\right. \\
& \left.+a_{15} d\left(g\left(\omega, \xi_{n}(\omega)\right), g\left(\omega, \xi_{n}(\omega)\right)\right)+a_{16} d\left(g\left(\omega, \rho_{n}(\omega)\right), g\left(\omega, \rho_{n}(\omega)\right)\right)\right] \\
& +a_{17} d\left(g\left(\omega, \gamma_{n-1}(\omega)\right), g\left(\omega, \gamma_{n}(\omega)\right)\right)+a_{18} d\left(g\left(\omega, \zeta_{n-1}(\omega)\right), g\left(\omega, \zeta_{n}(\omega)\right)\right) \\
& +a_{19} d\left(g\left(\omega, \xi_{n-1}(\omega)\right), g\left(\omega, \xi_{n}(\omega)\right)\right)+a_{20} d\left(g\left(\omega, \rho_{n-1}(\omega)\right), g\left(\omega, \rho_{n}(\omega)\right)\right) \\
& \preceq\left[a_{1} d\left(g\left(\omega, \gamma_{n-1}(\omega)\right), g\left(\omega, \gamma_{n}(\omega)\right)\right)+a_{2} d\left(g\left(\omega, \zeta_{n-1}(\omega)\right), g\left(\omega, \zeta_{n}(\omega)\right)\right)\right. \\
& +a_{3} d\left(g\left(\omega, \xi_{n-1}(\omega)\right), g\left(\omega, \xi_{n}(\omega)\right)\right)+a_{4} d\left(g\left(\omega, \rho_{n-1}(\omega)\right), g\left(\omega, \rho_{n}(\omega)\right)\right) \\
& +\left[a_{5} d\left(g\left(\omega, \gamma_{n}(\omega)\right), g\left(\omega, \gamma_{n+1}(\omega)\right)\right)+a_{6} d\left(g\left(\omega, \zeta_{n}(\omega)\right), g\left(\omega, \zeta_{n+1}(\omega)\right)\right)\right. \\
& \left.+a_{7} d\left(g\left(\omega, \xi_{n}(\omega)\right), g\left(\omega, \xi_{n+1}(\omega)\right)\right)+a_{8} d\left(g\left(\omega, \rho_{n}(\omega)\right), g\left(\omega, \rho_{n+1}(\omega)\right)\right)\right] \\
& +\left[\operatorname{sa}_{9}\left(d\left(g\left(\omega, \gamma_{n-1}(\omega)\right), g\left(\omega, \gamma_{n}(\omega)\right)\right)+d\left(g\left(\omega, \gamma_{n}(\omega)\right), g\left(\omega, \gamma_{n+1}(\omega)\right)\right)\right)\right. \\
& +\operatorname{sa}_{10}\left(\mathrm{~d}\left(\mathrm{~g}\left(\omega, \zeta_{n-1}(\omega)\right), g\left(\omega, \zeta_{n}(\omega)\right)\right)+\mathrm{d}\left(\mathrm{g}\left(\omega, \zeta_{n}(\omega)\right), \mathrm{g}\left(\omega, \zeta_{n+1}(\omega)\right)\right)\right) \\
& +\operatorname{sa}_{11}\left(d\left(g\left(\omega, \xi_{n-1}(\omega)\right), g\left(\omega, \xi_{n}(\omega)\right)\right)+d\left(g\left(\omega, \xi_{n}(\omega)\right), g\left(\omega, \xi_{n+1}(\omega)\right)\right)\right) \\
& \left.+\operatorname{sa}_{12}\left(d\left(g\left(\omega, \rho_{n-1}(\omega)\right), g\left(\omega, \rho_{\mathfrak{n}}(\omega)\right)\right)+d\left(g\left(\omega, \rho_{\mathfrak{n}}(\omega)\right), g\left(\omega, \rho_{n+1}(\omega)\right)\right)\right)\right]
\end{aligned}
$$




$$
\begin{aligned}
& +a_{17} d\left(g\left(\omega, \gamma_{n-1}(\omega)\right), g\left(\omega, \gamma_{n}(\omega)\right)\right)+a_{18} d\left(g\left(\omega, \zeta_{n-1}(\omega)\right), g\left(\omega, \zeta_{n}(\omega)\right)\right) \\
& +a_{19} d\left(g\left(\omega, \xi_{n-1}(\omega)\right), g\left(\omega, \xi_{n}(\omega)\right)\right)+a_{20} d\left(g\left(\omega, \rho_{n-1}(\omega)\right), g\left(\omega, \rho_{n}(\omega)\right)\right)
\end{aligned}
$$

Hence, we obtain that

$$
\begin{aligned}
& d\left(g\left(\omega, \gamma_{n}(\omega)\right), g\left(\omega, \gamma_{n+1}(\omega)\right)\right) \preceq\left(a_{1}+s a_{9}+a_{17}\right) d\left(g\left(\omega, \gamma_{n-1}(\omega)\right), g\left(\omega, \gamma_{n}(\omega)\right)\right) \\
& +\left(a_{2}+s a_{10}+a_{18}\right) d\left(g\left(\omega, \zeta_{n-1}(\omega)\right), g\left(\omega, \zeta_{n}(\omega)\right)\right) \\
& +\left(a_{3}+s a_{11}+a_{19}\right) d\left(g\left(\omega, \xi_{n-1}(\omega)\right), g\left(\omega, \xi_{n}(\omega)\right)\right) \\
& +\left(a_{4}+s a_{12}+a_{20}\right) d\left(g\left(\omega, \rho_{n-1}(\omega)\right), g\left(\omega, \rho_{n}(\omega)\right)\right) \\
& +\left(a_{5}+s a_{9}\right) d\left(g\left(\omega, \gamma_{n}(\omega)\right), g\left(\omega, \gamma_{n+1}(\omega)\right)\right) \\
& +\left(a_{6}+s a_{10}\right) d\left(g\left(\omega, \zeta_{n}(\omega)\right), g\left(\omega, \zeta_{n+1}(\omega)\right)\right) \\
& +\left(a_{7}+s a_{11}\right) d\left(g\left(\omega, \xi_{n}(\omega)\right), g\left(\omega, \xi_{n+1}(\omega)\right)\right) \\
& +\left(a_{8}+s a_{12}\right) d\left(g\left(\omega, \rho_{n}(\omega)\right), g\left(\omega, \rho_{n+1}(\omega)\right)\right) \text {. }
\end{aligned}
$$

Similarly, we can prove that

$$
\begin{aligned}
d\left(g\left(\omega, \zeta_{n}(\omega)\right), g\left(\omega, \zeta_{n+1}(\omega)\right)\right) \preceq & \left(a_{1}+s a_{9}+a_{17}\right) d\left(g\left(\omega, \zeta_{n-1}(\omega)\right), g\left(\omega, \zeta_{n}(\omega)\right)\right) \\
& +\left(a_{2}+s a_{10}+a_{18}\right) d\left(g\left(\omega, \xi_{n-1}(\omega)\right), g\left(\omega, \xi_{n}(\omega)\right)\right) \\
& +\left(a_{3}+s a_{11}+a_{19}\right) d\left(g\left(\omega, \rho_{n-1}(\omega)\right), g\left(\omega, \rho_{n}(\omega)\right)\right) \\
& +\left(a_{4}+s a_{12}+a_{20}\right) d\left(g\left(\omega, \gamma_{n-1}(\omega)\right), g\left(\omega, \gamma_{n}(\omega)\right)\right) \\
& +\left(a_{5}+s a_{9}\right) d\left(g\left(\omega, \zeta_{n}(\omega)\right), g\left(\omega, \zeta_{n+1}(\omega)\right)\right) \\
& +\left(a_{6}+s a_{10}\right) d\left(g\left(\omega, \xi_{n}(\omega)\right), g\left(\omega, \xi_{n+1}(\omega)\right)\right) \\
& +\left(a_{7}+s a_{11}\right) d\left(g\left(\omega, \rho_{n}(\omega)\right), g\left(\omega, \rho_{n+1}(\omega)\right)\right) \\
& +\left(a_{8}+s a_{12}\right) d\left(g\left(\omega, \gamma_{n}(\omega)\right), g\left(\omega, \gamma_{n+1}(\omega)\right)\right),
\end{aligned}
$$

and

$$
\begin{aligned}
d\left(g\left(\omega, \xi_{n}(\omega)\right), g\left(\omega, \xi_{n+1}(\omega)\right)\right) \preceq & \left(a_{1}+s a_{9}+a_{17}\right) d\left(g\left(\omega, \xi_{n-1}(\omega)\right), g\left(\omega, \xi_{n}(\omega)\right)\right) \\
& +\left(a_{2}+s a_{10}+a_{18}\right) d\left(g\left(\omega, \rho_{n-1}(\omega)\right), g\left(\omega, \rho_{n}(\omega)\right)\right) \\
& +\left(a_{3}+s a_{11}+a_{19}\right) d\left(g\left(\omega, \gamma_{n-1}(\omega)\right), g\left(\omega, \gamma_{n}(\omega)\right)\right) \\
& +\left(a_{4}+s a_{12}+a_{20}\right) d\left(g\left(\omega, \zeta_{n-1}(\omega)\right), g\left(\omega, \zeta_{n}(\omega)\right)\right) \\
& +\left(a_{5}+s a_{9}\right) d\left(g\left(\omega, \xi_{n}(\omega)\right), g\left(\omega, \xi_{n+1}(\omega)\right)\right) \\
& +\left(a_{6}+s a_{10}\right) d\left(g\left(\omega, \rho_{n}(\omega)\right), g\left(\omega, \rho_{n+1}(\omega)\right)\right) \\
& +\left(a_{7}+s a_{11}\right) d\left(g\left(\omega, \gamma_{n}(\omega)\right), g\left(\omega, \gamma_{n+1}(\omega)\right)\right) \\
& +\left(a_{8}+s a_{12}\right) d\left(g\left(\omega, \zeta_{n}(\omega)\right), g\left(\omega, \zeta_{n+1}(\omega)\right)\right),
\end{aligned}
$$

and

$$
\begin{aligned}
d\left(g\left(\omega, \rho_{n}(\omega)\right), g\left(\omega, \rho_{n+1}(\omega)\right)\right) \preceq & \left(a_{1}+s a_{9}+a_{17}\right) d\left(g\left(\omega, \rho_{n-1}(\omega)\right), g\left(\omega, \rho_{n}(\omega)\right)\right) \\
& +\left(a_{2}+s a_{10}+a_{18}\right) d\left(g\left(\omega, \gamma_{n-1}(\omega)\right), g\left(\omega, \gamma_{n}(\omega)\right)\right) \\
& +\left(a_{3}+s a_{11}+a_{19}\right) d\left(g\left(\omega, \zeta_{n-1}(\omega)\right), g\left(\omega, \zeta_{n}(\omega)\right)\right) \\
& +\left(a_{4}+s a_{12}+a_{20}\right) d\left(g\left(\omega, \xi_{n-1}(\omega)\right), g\left(\omega, \xi_{n}(\omega)\right)\right) \\
& +\left(a_{5}+s a_{9}\right) d\left(g\left(\omega, \rho_{n}(\omega)\right), g\left(\omega, \rho_{n+1}(\omega)\right)\right) \\
& +\left(a_{6}+s a_{10}\right) d\left(g\left(\omega, \gamma_{n}(\omega)\right), g\left(\omega, \gamma_{n+1}(\omega)\right)\right) \\
& +\left(a_{7}+s a_{11}\right) d\left(g\left(\omega, \zeta_{n}(\omega)\right), g\left(\omega, \zeta_{n+1}(\omega)\right)\right) \\
& +\left(a_{8}+s a_{12}\right) d\left(g\left(\omega, \xi_{n}(\omega)\right), g\left(\omega, \xi_{n+1}(\omega)\right)\right) .
\end{aligned}
$$


Put

$$
\begin{aligned}
d_{n}= & d\left(g\left(\omega, \gamma_{n}(\omega)\right), g\left(\omega, \gamma_{n+1}(\omega)\right)\right)+d\left(g\left(\omega, \zeta_{n}(\omega)\right), g\left(\omega, \zeta_{n+1}(\omega)\right)\right) \\
& +d\left(g\left(\omega, \xi_{n}(\omega)\right), g\left(\omega, \xi_{n+1}(\omega)\right)\right)+d\left(g\left(\omega, \rho_{n}(\omega)\right), g\left(\omega, \rho_{n+1}(\omega)\right)\right) .
\end{aligned}
$$

Uniting (3.2)-(3.5), ones assert that

$$
\begin{aligned}
d_{n} \preceq & \left(a_{1}+a_{2}+a_{3}+a_{4}+s a_{9}+s a_{10}+s a_{11}+s a_{12}+a_{17}+a_{18}+a_{19}+a_{20}\right) d_{n-1} \\
& +\left(a_{5}+a_{6}+a_{7}+a_{8}+s a_{9}+s a_{10}+s a_{11}+s a_{12}\right) d_{n} .
\end{aligned}
$$

\section{Furthermore,}

$$
\begin{aligned}
& \mathrm{d}\left(\mathrm{g}\left(\omega, \gamma_{\mathrm{n}+1}(\omega)\right), \mathrm{g}\left(\omega, \gamma_{\mathrm{n}}(\omega)\right)\right) \\
& =d\left(F\left(\omega,\left(\gamma_{n}(\omega), \zeta_{n}(\omega), \xi_{n}(\omega), \rho_{n}(\omega)\right)\right), F\left(\omega,\left(\gamma_{n-1}(\omega), \zeta_{n-1}(\omega), \xi_{n-1}(\omega), \rho_{n-1}(\omega)\right)\right)\right) \\
& \preceq\left[a _ { 1 } \mathrm { d } \left(\mathrm{g}\left(\omega, \gamma_{\mathrm{n}}(\omega)\right), \mathrm{F}\left(\omega,\left(\gamma_{\mathrm{n}}(\omega), \zeta_{\mathrm{n}}(\omega), \xi_{\mathrm{n}}(\omega), \rho_{\mathrm{n}}(\omega)\right)\right)\right.\right. \\
& +a_{2} d\left(g\left(\omega, \zeta_{n}(\omega)\right), F\left(\omega,\left(\zeta_{n}(\omega), \xi_{n}(\omega), \rho_{n}(\omega), \gamma_{n}(\omega)\right)\right)\right. \\
& +a_{3} d\left(g\left(\omega, \xi_{n}(\omega)\right), F\left(\omega,\left(\xi_{n}(\omega), \rho_{n}(\omega), \gamma_{n}(\omega), \zeta_{n}(\omega)\right)\right)\right. \\
& +a_{4} d\left(g\left(\omega, \rho_{n}(\omega)\right), F\left(\omega,\left(\rho_{n}(\omega), \gamma_{n}(\omega), \zeta_{n}(\omega), \xi_{n}(\omega)\right)\right)\right] \\
& +\left[a _ { 5 } d \left(g\left(\omega, \gamma_{n-1}(\omega)\right), F\left(\omega,\left(\gamma_{n-1}(\omega), \zeta_{n-1}(\omega), \xi_{n-1}(\omega), \rho_{n-1}(\omega)\right)\right)\right.\right. \\
& +a_{6} d\left(g\left(\omega, \zeta_{n-1}(\omega)\right), F\left(\omega,\left(\zeta_{n-1}(\omega), \xi_{n-1}(\omega), \rho_{n-1}(\omega), \gamma_{n-1}(\omega)\right)\right)\right. \\
& +a_{7} d\left(g\left(\omega, \xi_{n-1}(\omega)\right), F\left(\omega,\left(\xi_{n-1}(\omega), \rho_{n-1}(\omega), \gamma_{n-1}(\omega), \zeta_{n-1}(\omega)\right)\right)\right. \\
& +a_{8} d\left(g\left(\omega, \rho_{n-1}(\omega)\right), F\left(\omega,\left(\rho_{n-1}(\omega), \gamma_{n-1}(\omega), \zeta_{n-1}(\omega), \xi_{n-1}(\omega)\right)\right)\right] \\
& +\left[a _ { 9 } d \left(g\left(\omega, \gamma_{n}(\omega)\right), F\left(\omega,\left(\gamma_{n-1}(\omega), \zeta_{n-1}(\omega), \xi_{n-1}(\omega), \rho_{n-1}(\omega)\right)\right)\right.\right. \\
& +a_{10} d\left(g\left(\omega, \zeta_{n}(\omega)\right), F\left(\omega,\left(\zeta_{n-1}(\omega), \xi_{n-1}(\omega), \rho_{n-1}(\omega), \gamma_{n-1}(\omega)\right)\right)\right. \\
& +a_{11} d\left(g\left(\omega, \xi_{n}(\omega)\right), F\left(\omega,\left(\xi_{n-1}(\omega), \rho_{n-1}(\omega), \gamma_{n-1}(\omega), \zeta_{n-1}(\omega)\right)\right)\right. \\
& +a_{12} d\left(g\left(\omega, \rho_{n}(\omega)\right), F\left(\omega,\left(\rho_{n-1}(\omega), \gamma_{n-1}(\omega), \zeta_{n-1}(\omega), \xi_{n-1}(\omega)\right)\right)\right] \\
& +\left[a _ { 1 3 } d \left(g\left(\omega, \gamma_{n-1}(\omega)\right), F\left(\omega,\left(\gamma_{n}(\omega), \zeta_{n}(\omega), \xi_{n}(\omega), \rho_{n}(\omega)\right)\right)\right.\right. \\
& +a_{14} d\left(g\left(\omega, \zeta_{n-1}(\omega)\right), F\left(\omega,\left(\zeta_{n}(\omega), \xi_{n}(\omega), \rho_{n}(\omega), \gamma_{n}(\omega)\right)\right)\right. \\
& +a_{15} d\left(g\left(\omega, \xi_{n-1}(\omega)\right), F\left(\omega,\left(\xi_{n}(\omega), \rho_{n}(\omega), \gamma_{n}(\omega), \zeta_{n}(\omega)\right)\right)\right. \\
& \left.+a_{16} d\left(g\left(\omega, \rho_{n-1}(\omega)\right), F\left(\omega, \rho_{n}(\omega), \gamma_{n}(\omega), \zeta_{n}(\omega), \xi_{n}(\omega)\right)\right)\right] \\
& +\left[a_{17} d\left(g\left(\omega, \gamma_{n}(\omega)\right), g\left(\omega, \gamma_{n-1}(\omega)\right)\right)+a_{18} d\left(g\left(\omega, \zeta_{n}(\omega)\right), g\left(\omega, \zeta_{n-1}(\omega)\right)\right)\right. \\
& \left.+a_{19} d\left(g\left(\omega, \xi_{n}(\omega)\right), g\left(\omega, \xi_{n-1}(\omega)\right)\right)+a_{20} d\left(g\left(\omega, \rho_{n}(\omega)\right), g\left(\omega, \rho_{n-1}(\omega)\right)\right)\right] \\
& \preceq\left[a_{1} d\left(g\left(\omega, \gamma_{n}(\omega)\right), g\left(\omega, \gamma_{n+1}(\omega)\right)\right)+a_{2} d\left(g\left(\omega, \zeta_{n}(\omega)\right), g\left(\omega, \zeta_{n+1}(\omega)\right)\right)\right. \\
& +a_{3} d\left(g\left(\omega, \xi_{n}(\omega)\right), g\left(\omega, \xi_{n+1}(\omega)\right)\right)+a_{4} d\left(g\left(\omega, \rho_{n}(\omega)\right), g\left(\omega, \rho_{n+1}(\omega)\right)\right) \\
& +\left[a_{5} d\left(g\left(\omega, \gamma_{n-1}(\omega)\right), g\left(\omega, \gamma_{n}(\omega)\right)\right)+a_{6} d\left(g\left(\omega, \zeta_{n-1}(\omega)\right), g\left(\omega, \zeta_{n}(\omega)\right)\right)\right. \\
& \left.+a_{7} d\left(g\left(\omega, \xi_{n-1}(\omega)\right), g\left(\omega, \xi_{n}(\omega)\right)\right)+a_{8} d\left(g\left(\omega, \rho_{n-1}(\omega)\right), g\left(\omega, \rho_{n}(\omega)\right)\right)\right] \\
& +\left[a_{9} d\left(g\left(\omega, \gamma_{n}(\omega)\right), g\left(\omega, \gamma_{n}(\omega)\right)\right)+a_{10} d\left(g\left(\omega, \zeta_{n}(\omega)\right), g\left(\omega, \zeta_{n}(\omega)\right)\right)\right. \\
& \left.+a_{11} d\left(g\left(\omega, \xi_{n}(\omega)\right), g\left(\omega, \xi_{n}(\omega)\right)\right)+a_{12} d\left(g\left(\omega, \rho_{n}(\omega)\right), g\left(\omega, \rho_{n}(\omega)\right)\right)\right] \\
& +\left[a_{13} d\left(g\left(\omega, \gamma_{n-1}(\omega)\right), g\left(\omega, \gamma_{n+1}(\omega)\right)\right)+a_{14} d\left(g\left(\omega, \zeta_{n-1}(\omega)\right), g\left(\omega, \zeta_{n+1}(\omega)\right)\right)\right. \\
& \left.+a_{15} d\left(g\left(\omega, \xi_{n-1}(\omega)\right), g\left(\omega, \xi_{n+1}(\omega)\right)\right)+a_{16} d\left(g\left(\omega, \rho_{n-1}(\omega)\right), g\left(\omega, \rho_{n+1}(\omega)\right)\right)\right] \\
& +a_{17} d\left(g\left(\omega, \gamma_{n}(\omega)\right), g\left(\omega, \gamma_{n-1}(\omega)\right)\right)+a_{18} d\left(g\left(\omega, \zeta_{n}(\omega)\right), g\left(\omega, \zeta_{n-1}(\omega)\right)\right) \\
& +a_{19} d\left(g\left(\omega, \xi_{n}(\omega)\right), g\left(\omega, \xi_{n-1}(\omega)\right)\right)+a_{20} d\left(g\left(\omega, \rho_{n}(\omega)\right), g\left(\omega, \rho_{n-1}(\omega)\right)\right) \\
& \preceq\left[a_{1} d\left(g\left(\omega, \gamma_{n}(\omega)\right), g\left(\omega, \gamma_{n+1}(\omega)\right)\right)+a_{2} d\left(g\left(\omega, \zeta_{n}(\omega)\right), g\left(\omega, \zeta_{n+1}(\omega)\right)\right)\right. \\
& +a_{3} d\left(g\left(\omega, \xi_{n}(\omega)\right), g\left(\omega, \xi_{n+1}(\omega)\right)\right)+a_{4} d\left(g\left(\omega, \rho_{n}(\omega)\right), g\left(\omega, \rho_{n+1}(\omega)\right)\right) \\
& +\left[a_{5} d\left(g\left(\omega, \gamma_{n-1}(\omega)\right), g\left(\omega, \gamma_{n}(\omega)\right)\right)+a_{6} d\left(g\left(\omega, \zeta_{n-1}(\omega)\right), g\left(\omega, \zeta_{n}(\omega)\right)\right)\right. \\
& \left.+a_{7} d\left(g\left(\omega, \xi_{n-1}(\omega)\right), g\left(\omega, \xi_{n}(\omega)\right)\right)+a_{8} d\left(g\left(\omega, \rho_{n-1}(\omega)\right), g\left(\omega, \rho_{n}(\omega)\right)\right)\right] \\
& +\left[\operatorname{sa}_{13}\left(\mathrm{~d}\left(\mathrm{~g}\left(\omega, \gamma_{\mathrm{n}-1}(\omega)\right), \mathrm{g}\left(\omega, \gamma_{\mathrm{n}}(\omega)\right)\right)+\mathrm{d}\left(\mathrm{g}\left(\omega, \gamma_{\mathrm{n}}(\omega)\right), \mathrm{g}\left(\omega, \gamma_{\mathrm{n}+1}(\omega)\right)\right)\right)\right. \\
& +\operatorname{sa}_{14}\left(\mathrm{~d}\left(\mathrm{~g}\left(\omega, \zeta_{n-1}(\omega)\right), \mathrm{g}\left(\omega, \zeta_{n}(\omega)\right)\right)+\mathrm{d}\left(\mathrm{g}\left(\omega, \zeta_{n}(\omega)\right), \mathrm{g}\left(\omega, \zeta_{n+1}(\omega)\right)\right)\right)
\end{aligned}
$$




$$
\begin{aligned}
& +\operatorname{sa}_{15}\left(d\left(g\left(\omega, \xi_{n-1}(\omega)\right), g\left(\omega, \xi_{n}(\omega)\right)\right)+d\left(g\left(\omega, \xi_{n}(\omega)\right), g\left(\omega, \xi_{n+1}(\omega)\right)\right)\right) \\
& \left.+\operatorname{sa}_{16}\left(d\left(g\left(\omega, \rho_{n-1}(\omega)\right), g\left(\omega, \rho_{n}(\omega)\right)\right)+d\left(g\left(\omega, \rho_{n}(\omega)\right), g\left(\omega, \rho_{n+1}(\omega)\right)\right)\right)\right] \\
& +a_{17} d\left(g\left(\omega, \gamma_{n-1}(\omega)\right), g\left(\omega, \gamma_{n}(\omega)\right)\right)+a_{18} d\left(g\left(\omega, \zeta_{n-1}(\omega)\right), g\left(\omega, \zeta_{n}(\omega)\right)\right) \\
& +a_{19} d\left(g\left(\omega, \xi_{n-1}(\omega)\right), g\left(\omega, \xi_{n}(\omega)\right)\right)+a_{20} d\left(g\left(\omega, \rho_{n-1}(\omega)\right), g\left(\omega, \rho_{n}(\omega)\right)\right)
\end{aligned}
$$

Accordingly, it is clear that

$$
\begin{aligned}
d(g & \left.\left(\omega, \gamma_{n+1}(\omega)\right), g\left(\omega, \gamma_{n}(\omega)\right)\right) \\
\preceq & \left(a_{5}+s a_{13}+a_{17}\right) d\left(g\left(\omega, \gamma_{n-1}(\omega)\right), g\left(\omega, \gamma_{n}(\omega)\right)\right) \\
& +\left(a_{6}+s a_{14}+a_{18}\right) d\left(g\left(\omega, \zeta_{n-1}(\omega)\right), g\left(\omega, \zeta_{n}(\omega)\right)\right) \\
& +\left(a_{7}+s a_{15}+a_{19}\right) d\left(g\left(\omega, \xi_{n-1}(\omega)\right), g\left(\omega, \xi_{n}(\omega)\right)\right) \\
& +\left(a_{8}+s a_{16}+a_{20}\right) d\left(g\left(\omega, \rho_{n-1}(\omega)\right), g\left(\omega, \rho_{n}(\omega)\right)\right) \\
& +\left(a_{1}+s a_{13}\right) d\left(g\left(\omega, \gamma_{n}(\omega)\right), g\left(\omega, \gamma_{n+1}(\omega)\right)\right)+\left(a_{2}+s a_{14}\right) d\left(g\left(\omega, \zeta_{n}(\omega)\right), g\left(\omega, \zeta_{n+1}(\omega)\right)\right) \\
& +\left(a_{3}+s a_{15}\right) d\left(g\left(\omega, \xi_{n}(\omega)\right), g\left(\omega, \xi_{n+1}(\omega)\right)\right)+\left(a_{4}+s a_{16}\right) d\left(g\left(\omega, \rho_{n}(\omega)\right), g\left(\omega, \rho_{n+1}(\omega)\right)\right) .
\end{aligned}
$$

Similarly, we can prove that

$$
\begin{aligned}
\mathrm{d}\left(g\left(\omega, \zeta_{n+1}(\omega)\right), g\left(\omega, \zeta_{n}(\omega)\right)\right) \preceq & \left(a_{5}+s a_{13}+a_{17}\right) d\left(g\left(\omega, \zeta_{n-1}(\omega)\right), g\left(\omega, \zeta_{n}(\omega)\right)\right) \\
& +\left(a_{5}+s a_{14}+a_{18}\right) d\left(g\left(\omega, \xi_{n-1}(\omega)\right), g\left(\omega, \xi_{n}(\omega)\right)\right) \\
& +\left(a_{6}+s a_{15}+a_{19}\right) d\left(g\left(\omega, \rho_{n-1}(\omega)\right), g\left(\omega, \rho_{n}(\omega)\right)\right) \\
& +\left(a_{7}+s a_{16}+a_{20}\right) d\left(g\left(\omega, \gamma_{n-1}(\omega)\right), g\left(\omega, \gamma_{n}(\omega)\right)\right) \\
& +\left(a_{1}+s a_{13}\right) d\left(g\left(\omega, \zeta_{n}(\omega)\right), g\left(\omega, \zeta_{n+1}(\omega)\right)\right) \\
& +\left(a_{2}+s a_{14}\right) d\left(g\left(\omega, \xi_{n}(\omega)\right), g\left(\omega, \xi_{n+1}(\omega)\right)\right) \\
& +\left(a_{3}+s a_{15}\right) d\left(g\left(\omega, \rho_{n}(\omega)\right), g\left(\omega, \rho_{n+1}(\omega)\right)\right) \\
& +\left(a_{4}+s a_{16}\right) d\left(g\left(\omega, \gamma_{n}(\omega)\right), g\left(\omega, \gamma_{n+1}(\omega)\right)\right),
\end{aligned}
$$

and

$$
\begin{aligned}
d\left(g\left(\omega, \xi_{n+1}(\omega)\right), g\left(\omega, \xi_{n}(\omega)\right)\right) \preceq & \left(a_{5}+s a_{13}+a_{17}\right) d\left(g\left(\omega, \xi_{n-1}(\omega)\right), g\left(\omega, \xi_{n}(\omega)\right)\right) \\
& +\left(a_{6}+s a_{14}+a_{18}\right) d\left(g\left(\omega, \rho_{n-1}(\omega)\right), g\left(\omega, \rho_{n}(\omega)\right)\right) \\
& +\left(a_{7}+s a_{15}+a_{19}\right) d\left(g\left(\omega, \gamma_{n-1}(\omega)\right), g\left(\omega, \gamma_{n}(\omega)\right)\right) \\
& +\left(a_{8}+s a_{16}+a_{20}\right) d\left(g\left(\omega, \zeta_{n-1}(\omega)\right), g\left(\omega, \zeta_{n}(\omega)\right)\right) \\
& +\left(a_{1}+s a_{13}\right) d\left(g\left(\omega, \xi_{n}(\omega)\right), g\left(\omega, \xi_{n+1}(\omega)\right)\right) \\
& +\left(a_{2}+s a_{14}\right) d\left(g\left(\omega, \rho_{n}(\omega)\right), g\left(\omega, \rho_{n+1}(\omega)\right)\right) \\
& +\left(a_{3}+s a_{15}\right) d\left(g\left(\omega, \gamma_{n}(\omega)\right), g\left(\omega, \gamma_{n+1}(\omega)\right)\right) \\
& +\left(a_{4}+s a_{16}\right) d\left(g\left(\omega, \zeta_{n}(\omega)\right), g\left(\omega, \zeta_{n+1}(\omega)\right)\right),
\end{aligned}
$$

and

$$
\begin{aligned}
d\left(g\left(\omega, \rho_{n+1}(\omega)\right), g\left(\omega, \rho_{n}(\omega)\right)\right) \preceq & \left(a_{5}+s a_{13}+a_{17}\right) d\left(g\left(\omega, \rho_{n-1}(\omega)\right), g\left(\omega, \rho_{n}(\omega)\right)\right) \\
& +\left(a_{6}+s a_{14}+a_{18}\right) d\left(g\left(\omega, \gamma_{n-1}(\omega)\right), g\left(\omega, \gamma_{n}(\omega)\right)\right) \\
& +\left(a_{7}+s a_{15}+a_{19}\right) d\left(g\left(\omega, \zeta_{n-1}(\omega)\right), g\left(\omega, \zeta_{n}(\omega)\right)\right) \\
& +\left(a_{8}+s a_{16}+a_{20}\right) d\left(g\left(\omega, \xi_{n-1}(\omega)\right), g\left(\omega, \xi_{n}(\omega)\right)\right) \\
& +\left(a_{1}+s a_{13}\right) d\left(g\left(\omega, \rho_{n}(\omega)\right), g\left(\omega, \rho_{n+1}(\omega)\right)\right) \\
& +\left(a_{2}+s a_{14}\right) d\left(g\left(\omega, \gamma_{n}(\omega)\right), g\left(\omega, \gamma_{n+1}(\omega)\right)\right) \\
& +\left(a_{3}+s a_{15}\right) d\left(g\left(\omega, \zeta_{n}(\omega)\right), g\left(\omega, \zeta_{n+1}(\omega)\right)\right) \\
& +\left(a_{4}+s a_{16}\right) d\left(g\left(\omega, \xi_{n}(\omega)\right), g\left(\omega, \xi_{n+1}(\omega)\right)\right) .
\end{aligned}
$$


Uniting (3.7)-(3.10) one gets that

$$
\begin{aligned}
d_{n} \preceq & \left(a_{5}+a_{6}+a_{7}+a_{8}+s a_{13}+s a_{14}+s a_{15}+s a_{16}+a_{17}+a_{18}+a_{19}+a_{20}\right) d_{n-1} \\
& +\left(a_{1}+a_{2}+a_{3}+a_{4}+s a_{13}+s a_{14}+s a_{15}+s a_{16}\right) d_{n} .
\end{aligned}
$$

By using (3.6) and (3.11), it is easy to see that

$$
\begin{aligned}
2 d_{n} \preceq & \left(a_{1}+a_{2}+a_{3}+a_{4}+a_{5}+a_{6}+a_{7}+a_{8}+s a_{9}+s a_{10}+s a_{11}+s a_{12}+s a_{13}+s a_{14}+s a_{15}\right. \\
& \left.+s a_{16}+2\left(a_{17}+a_{18}+a_{19}+a_{20}\right)\right) d_{n-1}+\left(a_{1}+a_{2}+a_{3}+a_{4}+a_{5}+a_{6}+a_{7}+a_{8}+s a_{10}\right. \\
& \left.+s a_{11}+s a_{12}+s a_{13}+s a_{14}+s a_{15}+s a_{16}\right) d_{n} .
\end{aligned}
$$

Put $k_{1}=a_{17}+a_{18}+a_{19}+a_{20}$ and $k=\sum_{i=1}^{8} a_{i}+\sum_{i=9}^{16} s a_{i}$, then

$$
(2 e-k) d_{n} \preceq\left(2 k_{1}+k\right) d_{n-1} .
$$

Because of $(s+1) \rho\left(\sum_{i=1}^{8} a_{i}\right)+s(s+1) \rho\left(\sum_{i=9}^{16} s a_{i}\right)+2 s \rho\left(k_{1}\right)<2$ and $s \geqslant 1$, it is clear that

$$
\rho\left(\sum_{i=1}^{8} a_{i}+\sum_{i=9}^{16} s a_{i}\right) \leqslant \rho\left(\sum_{i=1}^{8} a_{i}\right)+s \rho\left(\sum_{i=9}^{16} s a_{i}\right)<2 .
$$

Then by Lemma 2.4 and Lemma 2.5 , it follows that $2 e-k$ is invertible. Furthermore, $(2 e-k)^{-1}=$ $\sum_{i=0}^{\infty} \frac{k^{i}}{2^{i+1}}$. Multiplying in both sides of (3.12) by $(2 e-k)^{-1}$, we obtain

$$
d_{n} \preceq(2 e-k)^{-1}\left(2 k_{1}+k\right) d_{n-1} .
$$

Denote $h=(2 e-k)^{-1}\left(2 k_{1}+k\right)$, then by (3.13) we get

$$
d_{n} \preceq h d_{n-1} \preceq \cdots \preceq h^{n} d_{0} .
$$

Note by Lemma 2.4 that

$$
\rho\left(\sum_{i=0}^{n} \frac{k^{i}}{2^{i+1}}\right) \leqslant \sum_{i=0}^{n} \rho\left(\frac{k^{i}}{2^{i+1}}\right) \leqslant \sum_{i=0}^{n}\left(\frac{\left[\rho\left(k^{i}\right)\right]}{2^{i+1}}\right),
$$

so by Lemma 2.8 it leads to

$$
\rho\left(\sum_{i=0}^{\infty} \frac{k^{i}}{2^{i+1}}\right) \leqslant \sum_{i=0}^{\infty}\left(\frac{\left[\rho\left(k^{i}\right)\right]}{2^{i+1}}\right) .
$$

Because $a_{i} a_{j}=a_{j} a_{i}(i, j=1, \ldots, 20)$ implies $k_{1}$ commutes $k$, we have

$$
\begin{aligned}
(2 e-k)^{-1}\left(2 k_{1}+k\right) & =\left(\sum_{i=0}^{\infty} \frac{k^{i}}{2^{i+1}}\right)\left(2 k_{1}+k\right) \\
& =2\left(\sum_{i=0}^{\infty} \frac{k^{i}}{2^{i+1}}\right) k_{1}+\left(\sum_{i=0}^{\infty} \frac{k^{i}}{2^{i+1}}\right) k \\
& =2 k_{1}\left(\sum_{i=0}^{\infty} \frac{k^{i}}{2^{i+1}}\right)+k\left(\sum_{i=0}^{\infty} \frac{k^{i}}{2^{i+1}}\right) \\
& =\left(2 k_{1}+k\right)\left(\sum_{i=0}^{\infty} \frac{k^{i}}{2^{i+1}}\right) \\
& =\left(2 k_{1}+k\right)(2 e-k)^{-1},
\end{aligned}
$$


that is to say, $(2 e-k)^{-1}$ commutes with $2 k_{1}+k$. Note that $(s+1) \rho\left(\sum_{i=1}^{8} a_{i}\right)+s(s+1) \rho\left(\sum_{i=9}^{16} s a_{i}\right)+$ $2 s \rho\left(k_{1}\right)<2$ means $2 s \rho\left(k_{1}\right)+(s+1) \rho(k)<2$, then by Lemma 2.5 we again

$$
\begin{aligned}
\rho(h) & =\rho\left((2 e-k)^{-1}\left(2 k_{1}+k\right)\right) \\
& \leqslant \rho\left((2 e-k)^{-1}\right) \rho\left(2 k_{1}+k\right) \\
& \leqslant \rho\left(\sum_{i=0}^{\infty} \frac{k^{i}}{2^{i+1}}\right)\left(2 \rho\left(k_{1}+\rho(k)\right)\right) \\
& \leqslant \sum_{i=0}^{n}\left(\frac{\left[\rho\left(k^{i}\right)\right]}{2^{i+1}}\right)\left(2 \rho\left(k_{1}+\rho(k)\right)\right) \\
& \leqslant \frac{1}{2-\rho(k)}\left(2 \rho\left(k_{1}+\rho(k)\right)\right)<\frac{1}{s} \leqslant 1,
\end{aligned}
$$

which establishes that $e-h$ is invertible and $\left\|h^{n}\right\| \rightarrow 0$ as $n \rightarrow \infty$. Thus for all $m>n \geqslant 1$, one has

$$
\begin{aligned}
& d\left(g\left(\omega, \gamma_{n}(\omega)\right), g\left(\omega, \gamma_{m}(\omega)\right)\right) \preceq \operatorname{sd}\left(g\left(\omega, \gamma_{n}(\omega)\right), g\left(\omega, \gamma_{n+1}(\omega)\right)\right) \\
& +s^{2} d\left(g\left(\omega, \gamma_{n+1}(\omega)\right), g\left(\omega, \gamma_{n+2}(\omega)\right)\right) \\
& \text { : } \\
& +s^{m-n} d\left(g\left(\omega, \gamma_{m-1}(\omega)\right), g\left(\omega, \gamma_{m}(\omega)\right)\right), \\
& \mathrm{d}\left(\mathrm{g}\left(\omega, \zeta_{\mathrm{n}}(\omega)\right), \mathrm{g}\left(\omega, \zeta_{\mathrm{m}}(\omega)\right)\right) \preceq \operatorname{sd}\left(\mathrm{g}\left(\omega, \zeta_{\mathrm{n}}(\omega)\right), \mathrm{g}\left(\omega, \zeta_{\mathrm{n}+1}(\omega)\right)\right) \\
& +s^{2} d\left(g\left(\omega, \zeta_{n+1}(\omega)\right), g\left(\omega, \zeta_{n+2}(\omega)\right)\right) \\
& \vdots \\
& +s^{m-n} d\left(g\left(\omega, \zeta_{m-1}(\omega)\right), g\left(\omega, \zeta_{m}(\omega)\right)\right), \\
& d\left(g\left(\omega, \xi_{\mathfrak{n}}(\omega)\right), g\left(\omega, \xi_{m}(\omega)\right)\right) \preceq \operatorname{sd}\left(g\left(\omega, \xi_{\mathfrak{n}}(\omega)\right), g\left(\omega, \xi_{n+1}(\omega)\right)\right) \\
& +s^{2} d\left(g\left(\omega, \xi_{n+1}(\omega)\right), g\left(\omega, \xi_{n+2}(\omega)\right)\right) \\
& \text { : } \\
& +s^{m-n} d\left(g\left(\omega, \xi_{m-1}(\omega)\right), g\left(\omega, \xi_{m}(\omega)\right)\right), \\
& d\left(g\left(\omega, \rho_{n}(\omega)\right), g\left(\omega, \rho_{m}(\omega)\right)\right) \preceq \operatorname{sd}\left(g\left(\omega, \rho_{n}(\omega)\right), g\left(\omega, \rho_{n+1}(\omega)\right)\right) \\
& +s^{2} d\left(g\left(\omega, \rho_{n+1}(\omega)\right), g\left(\omega, \rho_{n+2}(\omega)\right)\right) \\
& \vdots \\
& +s^{m-n} d\left(g\left(\omega, \rho_{m-1}(\omega)\right), g\left(\omega, \rho_{m}(\omega)\right)\right) .
\end{aligned}
$$

Now, by (3.14) and $s \rho(h)<1$, it follows that

$$
\begin{aligned}
d(g & \left.\left(\omega, \gamma_{n}(\omega)\right), g\left(\omega, \gamma_{m}(\omega)\right)\right)+d\left(g\left(\omega, \zeta_{n}(\omega)\right), g\left(\omega, \zeta_{m}(\omega)\right)\right) \\
& +d\left(g\left(\omega, \xi_{n}(\omega)\right), g\left(\omega, \xi_{m}(\omega)\right)\right)+d\left(g\left(\omega, \rho_{n}(\omega)\right), g\left(\omega, \rho_{m}(\omega)\right)\right) \\
& \preceq s d_{n}+s^{2} d_{n+1}+\cdots+s^{m-n} d_{m-1} \\
& \preceq s h^{n} d_{0}+s^{2} h^{n+1} d_{0}+\cdots+s^{m-n} h^{m-1} d_{0} \\
& =\left(s h^{n}+s^{2} h^{n+1}+\cdots+s^{m-n} h^{m-1}\right) d_{0} \\
& =s^{n}\left(e+s h+(s h)^{2}+\cdots+(s h)^{m-n-1}\right) d_{0} \\
& \preceq(e-s h)^{-1} s^{n} d_{0} .
\end{aligned}
$$

Owing to

$$
\left\|(e-s h)^{-1} \operatorname{sh}^{n} d_{0}\right\| \leqslant\left\|(e-s h)^{-1}\right\| s\left\|h^{n}\right\|\left\|d_{0}\right\| \rightarrow 0,(n \rightarrow \infty),
$$


we have $e-\operatorname{sh}^{-1} \operatorname{sh}^{n} d_{0} \rightarrow 0(n \rightarrow \infty)$. According to Lemma 2.6, and for any $c \gg \theta$, there exists $N_{0}$ such that for all $n>N_{0},(e-s h)^{-1} s h^{n} d_{0} \ll c$. Furthermore, from (3.15) and for any $m>n>N_{0}$, it follows that

$$
\begin{aligned}
& d\left(g\left(\omega, \gamma_{n}(\omega)\right), g\left(\omega, \gamma_{m}(\omega)\right)\right)+d\left(g\left(\omega, \zeta_{n}(\omega)\right), g\left(\omega, \zeta_{m}(\omega)\right)\right) \\
& \quad+d\left(g\left(\omega, \xi_{n}(\omega)\right), g\left(\omega, \xi_{m}(\omega)\right)\right)+d\left(g\left(\omega, \rho_{n}(\omega)\right), g\left(\omega, \rho_{m}(\omega)\right)\right) \ll c,
\end{aligned}
$$

which implies that

$$
\begin{array}{ll}
d\left(g\left(\omega, \gamma_{n}(\omega)\right), g\left(\omega, \gamma_{m}(\omega)\right)\right) \ll c, & d\left(g\left(\omega, \zeta_{n}(\omega)\right), g\left(\omega, \zeta_{m}(\omega)\right)\right) \ll c, \\
d\left(g\left(\omega, \xi_{n}(\omega)\right), g\left(\omega, \xi_{m}(\omega)\right)\right) \ll c, & d\left(g\left(\omega, \rho_{n}(\omega)\right), g\left(\omega, \rho_{m}(\omega)\right)\right) \ll c .
\end{array}
$$

Hence, $\left\{g\left(\omega, \gamma_{n}(\omega)\right)\right\},\left\{g\left(\omega, \zeta_{n}(\omega)\right)\right\},\left\{g\left(\omega, \xi_{n}(\omega)\right)\right\},\left\{g\left(\omega, \rho_{n}(\omega)\right)\right\}$ are Cauchy sequences in $g(X)$. Since $\mathrm{g}(X)$ is complete, there exist $\gamma^{*}(\omega), \zeta^{*}(\omega), \xi^{*}(\omega)$ and $\rho^{*}(\omega) \in X$ for all $\omega \in \Omega$ such that

$$
\begin{array}{ll}
g\left(\omega,\left(\gamma_{n}(\omega)\right)\right) \rightarrow g\left(\omega,\left(\gamma^{*}(\omega)\right)\right), & g\left(\omega,\left(\zeta_{n}(\omega)\right)\right) \rightarrow g\left(\omega,\left(\zeta^{*}(\omega)\right)\right), \\
g\left(\omega,\left(\xi_{n}(\omega)\right)\right) \rightarrow g\left(\omega,\left(\xi^{*}(\omega)\right)\right), & g\left(\omega,\left(\rho_{n}(\omega)\right)\right) \rightarrow g\left(\omega,\left(\rho^{*}(\omega)\right)\right),
\end{array}
$$

as $\mathrm{n} \rightarrow \infty$. Moreover, note that

$$
\begin{aligned}
& \mathrm{d}\left(\mathrm{F}\left(\omega,\left(\gamma^{*}(\omega), \zeta^{*}(\omega), \xi^{*}(\omega), \rho^{*}(\omega)\right)\right), g\left(\omega, \gamma^{*}(\omega)\right)\right) \\
& \preceq \mathrm{s}\left(\mathrm{d}\left(\mathrm{F}\left(\omega,\left(\gamma^{*}(\omega), \zeta^{*}(\omega), \xi^{*}(\omega), \rho^{*}(\omega)\right)\right), \mathrm{g}\left(\omega, \gamma_{n+1}(\omega)\right)\right)+\mathrm{d}\left(\mathrm{g}\left(\omega, \gamma_{n+1}(\omega)\right), \mathrm{g}\left(\omega, \gamma^{*}(\omega)\right)\right)\right) \\
& =s\left(d\left(F\left(\omega,\left(\gamma^{*}(\omega), \zeta^{*}(\omega), \xi^{*}(\omega), \rho^{*}(\omega)\right)\right), F\left(\omega,\left(\gamma_{n}(\omega), \zeta_{n}(\omega), \xi_{n}(\omega), \rho_{n}(\omega)\right)\right)\right)\right. \\
& \left.+\operatorname{sd}\left(g\left(\omega, \gamma_{n+1}(\omega)\right), g\left(\omega, \gamma^{*}(\omega)\right)\right)\right) \\
& \preceq s\left[a _ { 1 } d \left(g\left(\omega, \gamma^{*}(\omega)\right), F\left(\omega,\left(\gamma^{*}(\omega), \zeta^{*}(\omega), \xi^{*}(\omega), \rho^{*}(\omega)\right)\right)\right.\right. \\
& +a_{2} d\left(g\left(\omega, \zeta^{*}(\omega)\right), F\left(\omega,\left(\zeta^{*}(\omega), \xi^{*}(\omega), \rho^{*}(\omega), \gamma^{*}(\omega)\right)\right)\right. \\
& +a_{3} d\left(g\left(\omega, \xi^{*}(\omega)\right), F\left(\omega,\left(\xi^{*}(\omega), \rho^{*}(\omega), \gamma^{*}(\omega), \zeta^{*}(\omega)\right)\right)\right. \\
& +a_{4} d\left(g\left(\omega, \rho^{*}(\omega)\right), F\left(\omega,\left(\rho^{*}(\omega), \gamma^{*}(\omega), \zeta^{*}(\omega), \xi^{*}(\omega)\right)\right)\right] \\
& +s\left[a _ { 5 } d \left(g\left(\omega, \gamma_{n}(\omega)\right), F\left(\omega,\left(\gamma_{n}(\omega), \zeta_{n}(\omega), \xi_{n}(\omega), \rho_{n}(\omega)\right)\right)\right.\right. \\
& +a_{6} \mathrm{~d}\left(\mathrm{~g}\left(\omega, \zeta_{n}(\omega)\right), \mathrm{F}\left(\omega,\left(\zeta_{n}(\omega), \xi_{n}(\omega), \rho_{n}(\omega), \gamma_{n}(\omega)\right)\right)\right. \\
& +a_{7} d\left(g\left(\omega, \xi_{n}(\omega)\right), F\left(\omega,\left(\xi_{n}(\omega), \rho_{n}(\omega), \gamma_{n}(\omega), \zeta_{n}(\omega)\right)\right)\right. \\
& +a_{8} d\left(g\left(\omega, \rho_{n}(\omega)\right), F\left(\omega,\left(\rho_{n}(\omega), \gamma_{n}(\omega), \zeta_{n}(\omega), \xi_{n}(\omega)\right)\right)\right] \\
& +s\left[a _ { 9 } d \left(g\left(\omega, \gamma^{*}(\omega)\right), F\left(\omega,\left(\gamma_{n}(\omega), \zeta_{n}(\omega), \xi_{n}(\omega), \rho_{n}(\omega)\right)\right)\right.\right. \\
& +a_{10} d\left(g\left(\omega, \zeta^{*}(\omega)\right), F\left(\omega,\left(\zeta_{n}(\omega), \xi_{n}(\omega), \rho_{n}(\omega), \gamma_{n}(\omega)\right)\right)\right. \\
& +a_{11} d\left(g\left(\omega, \xi^{*}(\omega)\right), F\left(\omega,\left(\xi_{n}(\omega), \rho_{n}(\omega), \gamma_{n}(\omega), \zeta_{n}(\omega)\right)\right)\right. \\
& +a_{12} d\left(g\left(\omega, \rho^{*}(\omega)\right), F\left(\omega,\left(\rho_{n}(\omega), \gamma_{n}(\omega), \zeta_{n}(\omega), \xi_{n}(\omega)\right)\right)\right] \\
& +s\left[a _ { 1 3 } d \left(g\left(\omega, \gamma_{n}(\omega)\right), F\left(\omega,\left(\gamma^{*}(\omega), \zeta^{*}(\omega), \xi^{*}(\omega), \rho^{*}(\omega)\right)\right)\right.\right. \\
& +a_{14} d\left(g\left(\omega, \zeta_{n}(\omega)\right), F\left(\omega,\left(\zeta^{*}(\omega), \xi^{*}(\omega), \rho^{*}(\omega), \gamma^{*}(\omega)\right)\right)\right. \\
& +a_{15} d\left(g\left(\omega, \xi_{n}(\omega)\right), F\left(\omega,\left(\xi^{*} \omega\right), \rho^{*}(\omega), \gamma^{*}(\omega), \zeta^{*}(\omega)\right)\right) \\
& +a_{16} d\left(g\left(\omega, \rho_{n}(\omega)\right), F\left(\omega,\left(\rho^{*}(\omega), \gamma^{*}(\omega), \zeta^{*}(\omega), \xi^{*}(\omega)\right)\right)\right] \\
& +s\left[a_{17} d\left(g\left(\omega, \gamma^{*}(\omega)\right), g\left(\omega, \gamma_{n}(\omega)\right)\right)+a_{18} d\left(g\left(\omega, \zeta^{*}(\omega)\right), g\left(\omega, \zeta_{n}(\omega)\right)\right)\right. \\
& \left.+a_{19} d\left(g\left(\omega, \xi^{*}(\omega)\right), g\left(\omega, \xi_{n}(\omega)\right)\right)+a_{20} d\left(g\left(\omega, \rho^{*}(\omega)\right), g\left(\omega, \rho_{n}(\omega)\right)\right)\right] \\
& \left.+\operatorname{sd}\left(g\left(\omega, \gamma_{n+1}(\omega)\right), g\left(\omega, \gamma^{*}(\omega)\right)\right)\right) \\
& \preceq \mathrm{s}\left[\mathrm { a } _ { 1 } \mathrm { d } \left(\mathrm{g}\left(\omega, \gamma^{*}(\omega)\right), \mathrm{F}\left(\omega,\left(\gamma^{*}(\omega), \zeta^{*}(\omega), \xi^{*}(\omega), \rho^{*}(\omega)\right)\right)\right.\right. \\
& +a_{2} d\left(g\left(\omega, \zeta^{*}(\omega)\right), F\left(\omega,\left(\zeta^{*}(\omega), \xi^{*}(\omega), \rho^{*}(\omega), \gamma^{*}(\omega)\right)\right)\right. \\
& +a_{3} d\left(g\left(\omega, \xi^{*}(\omega)\right), F\left(\omega,\left(\xi^{*}(\omega), \rho^{*}(\omega), \gamma^{*}(\omega), \zeta^{*}(\omega)\right)\right)\right. \\
& +a_{4} d\left(g\left(\omega, \rho^{*}(\omega)\right), F\left(\omega,\left(\rho^{*}(\omega), \gamma^{*}(\omega), \zeta^{*}(\omega), \xi^{*}(\omega)\right)\right)\right] \\
& +s\left[\operatorname{sa}_{5} d\left(g\left(\omega, \gamma_{n}(\omega)\right), g\left(\omega, \gamma^{*}(\omega)\right)\right)+s_{5} d\left(g\left(\omega, \gamma^{*}(\omega)\right), g\left(\omega, \gamma_{n+1}(\omega)\right)\right)\right. \\
& +\operatorname{sa}_{6} \mathrm{~d}\left(\mathrm{~g}\left(\omega, \zeta_{n}(\omega)\right), g\left(\omega, \zeta^{*}(\omega)\right)\right)+\operatorname{sa}_{6} \mathrm{~d}\left(\mathrm{~g}\left(\omega, \zeta^{*}(\omega)\right), g\left(\omega, \zeta_{n+1}(\omega)\right)\right)
\end{aligned}
$$


$+s a_{7} d\left(g\left(\omega, \xi_{n}(\omega)\right), g\left(\omega, \xi^{*}(\omega)\right)\right)+s a_{7} d\left(g\left(\omega, \xi^{*}(\omega)\right), g\left(\omega, \xi_{n+1}(\omega)\right)\right)$

$\left.+s a_{8} d\left(g\left(\omega, \rho_{n}(\omega)\right), g\left(\omega, \rho^{*}(\omega)\right)\right)+s a_{8} d\left(g\left(\omega, \rho^{*}(\omega)\right), g\left(\omega, \rho_{n+1}(\omega)\right)\right)\right]$

$+s\left[a_{9} d\left(g\left(\omega, \gamma^{*}(\omega)\right), g\left(\omega, \gamma_{n+1}(\omega)\right)\right)+a_{10} d\left(g\left(\omega, \zeta^{*}(\omega)\right), g\left(\omega, \zeta_{n+1}(\omega)\right)\right)\right.$

$\left.+a_{11} d\left(g\left(\omega, \xi^{*}(\omega)\right), g\left(\omega, \xi_{n+1}(\omega)\right)\right)+a_{12} d\left(g\left(\omega, \rho^{*}(\omega)\right), g\left(\omega, \rho_{n+1}(\omega)\right)\right)\right]$

$+s\left[\operatorname{sa}_{13} \mathrm{~d}\left(\mathrm{~g}\left(\omega, \gamma_{\mathrm{n}}(\omega)\right), \mathrm{g}\left(\omega, \gamma^{*}(\omega)\right)\right)+\operatorname{sa}_{13} \mathrm{~d}\left(\mathrm{~g}\left(\omega, \gamma^{*}(\omega)\right), \mathrm{F}\left(\omega,\left(\gamma^{*}(\omega), \zeta^{*}(\omega), \xi^{*}(\omega), \rho^{*}(\omega)\right)\right)\right.\right.$

$+\operatorname{sa}_{14} \mathrm{~d}\left(\mathrm{~g}\left(\omega, \zeta_{n}(\omega)\right), g\left(\omega, \zeta^{*}(\omega)\right)\right)+\operatorname{sa}_{14} \mathrm{~d}\left(\mathrm{~g}\left(\omega, \zeta^{*}(\omega)\right), \mathrm{F}\left(\omega,\left(\zeta^{*}(\omega), \xi^{*}(\omega), \rho^{*}(\omega), \gamma^{*}(\omega)\right)\right)\right.$

$+\operatorname{sa}_{15} d\left(g\left(\omega, \xi_{n}(\omega)\right), g\left(\omega, \xi^{*}(\omega)\right)\right)+s a_{15} d\left(g\left(\omega, \xi^{*}(\omega)\right), F\left(\omega,\left(\xi^{*}(\omega), \rho^{*}(\omega), \gamma^{*}(\omega), \zeta^{*}(\omega)\right)\right)\right.$

$+\operatorname{sa}_{16} \mathrm{~d}\left(\mathrm{~g}\left(\omega, \rho_{\mathrm{n}}(\omega)\right), \mathrm{g}\left(\omega, \rho^{*}(\omega)\right)\right)+\operatorname{sa}_{16} \mathrm{~d}\left(\mathrm{~g}\left(\omega, \rho^{*}(\omega)\right), \mathrm{F}\left(\omega,\left(\rho^{*}(\omega), \gamma^{*}(\omega), \zeta^{*}(\omega), \xi^{*}(\omega)\right)\right)\right]$

$+s\left[a_{17} d\left(g\left(\omega, \gamma^{*}(\omega)\right), g\left(\omega, \gamma_{n}(\omega)\right)\right)+a_{18} d\left(g\left(\omega, \zeta^{*}(\omega)\right), g\left(\omega, \zeta_{n}(\omega)\right)\right)\right.$

$\left.+a_{19} d\left(g\left(\omega, \xi^{*}(\omega)\right), g\left(\omega, \xi_{n}(\omega)\right)\right)+a_{20} d\left(g\left(\omega, \rho^{*}(\omega)\right), g\left(\omega, \rho_{n}(\omega)\right)\right)\right]$

$\left.+\operatorname{sd}\left(g\left(\omega, \gamma_{n+1}(\omega)\right), g\left(\omega, \gamma^{*}(\omega)\right)\right)\right)$.

Hence,

$$
\begin{aligned}
d(F(\omega) & \left.\left.\left(\gamma^{*}(\omega), \zeta^{*}(\omega), \xi^{*}(\omega), \rho^{*}(\omega)\right)\right), g\left(\omega, \gamma^{*}(\omega)\right)\right) \\
\preceq & \left(s a_{1}+s^{2} a_{13}\right) d\left(g\left(\omega, \gamma^{*}(\omega)\right), F\left(\omega,\left(\gamma^{*}(\omega), \zeta^{*}(\omega), \xi^{*}(\omega), \rho^{*}(\omega)\right)\right)\right) \\
& +\left(s a_{2}+s^{2} a_{14}\right) d\left(g\left(\omega, \zeta^{*}(\omega)\right), F\left(\omega,\left(\zeta^{*}(\omega), \xi^{*}(\omega), \rho^{*}(\omega), \gamma^{*}(\omega)\right)\right)\right) \\
& +\left(s a_{3}+s^{2} a_{15}\right) d\left(g\left(\omega, \xi^{*}(\omega)\right), F\left(\omega,\left(\xi^{*}(\omega), \rho^{*}(\omega), \gamma^{*}(\omega), \zeta^{*}(\omega)\right)\right)\right) \\
& +\left(s a_{4}+s^{2} a_{16}\right) d\left(g\left(\omega, \rho^{*}(\omega)\right), F\left(\omega,\left(\rho^{*}(\omega), \gamma^{*}(\omega), \zeta^{*}(\omega), \xi^{*}(\omega)\right)\right)\right) \\
& +\left(s^{2} a_{5}+s^{2} a_{13}+s a_{17}\right) d\left(g\left(\omega, \gamma^{*}(\omega)\right), g\left(\omega, \gamma_{n}(\omega)\right)\right) \\
& +\left(s^{2} a_{6}+s^{2} a_{14}+s a_{18}\right) d\left(g\left(\omega, \zeta^{*}(\omega)\right), g\left(\omega, \zeta_{n}(\omega)\right)\right) \\
& +\left(s^{2} a_{7}+s^{2} a_{15}+s a_{19}\right) d\left(g\left(\omega, \xi^{*}(\omega)\right), g\left(\omega, \xi_{n}(\omega)\right)\right) \\
& +\left(s^{2} a_{8}+s^{2} a_{16}+s a_{20}\right) d\left(g\left(\omega, \rho^{*}(\omega)\right), g\left(\omega, \rho_{n}(\omega)\right)\right) \\
& +\left(s^{2} a_{5}+s a_{9}+s\right) d\left(g\left(\omega, \gamma^{*}(\omega)\right), g(\omega, \gamma, \gamma+1(\omega))\right) \\
& +\left(s^{2} a_{6}+s a_{10}\right) d\left(g\left(\omega, \zeta^{*}(\omega)\right), g\left(\omega, \zeta_{n+1}(\omega)\right)\right) \\
& +\left(s^{2} a_{7}+s a_{11}\right) d\left(g\left(\omega, \xi^{*}(\omega)\right), g\left(\omega, \xi_{n+1}(\omega)\right)\right) \\
& +\left(s^{2} a_{8}+s a_{12}\right) d\left(g\left(\omega, \rho^{*}(\omega)\right), g\left(\omega, \rho_{n+1}(\omega)\right)\right) .
\end{aligned}
$$

Similarly,

$$
\begin{aligned}
d & \left.F\left(\omega,\left(\zeta^{*}(\omega), \xi^{*}(\omega), \rho^{*}(\omega), \gamma^{*}(\omega)\right)\right), g\left(\omega, \zeta^{*}(\omega)\right)\right) \\
\preceq & \left(s a_{1}+s^{2} a_{13}\right) d\left(g\left(\omega, \zeta^{*}(\omega)\right), F\left(\omega,\left(\zeta^{*}(\omega), \xi^{*}(\omega), \rho^{*}(\omega), \gamma^{*}(\omega)\right)\right)\right) \\
& +\left(s a_{2}+s^{2} a_{14}\right) d\left(g\left(\omega, \xi^{*}(\omega)\right), F\left(\omega,\left(\xi^{*}(\omega), \rho^{*}(\omega), \gamma^{*}(\omega), \zeta^{*}(\omega)\right)\right)\right) \\
& +\left(s a_{3}+s^{2} a_{15}\right) d\left(g\left(\omega, \rho^{*}(\omega)\right), F\left(\omega,\left(\rho^{*}(\omega), \gamma^{*}(\omega), \zeta^{*}(\omega), \xi^{*}(\omega)\right)\right)\right) \\
& +\left(s a_{4}+s^{2} a_{16}\right) d\left(g\left(\omega, \gamma^{*}(\omega)\right), F\left(\omega,\left(\gamma^{*}(\omega), \zeta^{*}(\omega), \xi^{*}(\omega), \rho^{*}(\omega)\right)\right)\right) \\
& +\left(s^{2} a_{5}+s^{2} a_{13}+s a_{17}\right) d\left(g\left(\omega, \zeta^{*}(\omega)\right), g\left(\omega, \zeta_{n}(\omega)\right)\right) \\
& +\left(s^{2} a_{6}+s^{2} a_{14}+s a_{18}\right) d\left(g\left(\omega, \xi^{*}(\omega)\right), g\left(\omega, \xi_{n}(\omega)\right)\right) \\
& +\left(s^{2} a_{7}+s^{2} a_{15}+s a_{19}\right) d\left(g\left(\omega, \rho^{*}(\omega)\right), g\left(\omega, \rho_{n}(\omega)\right)\right) \\
& +\left(s^{2} a_{8}+s^{2} a_{16}+s a_{20}\right) d\left(g\left(\omega, \gamma^{*}(\omega)\right), g\left(\omega, \gamma_{n}(\omega)\right)\right) \\
& +\left(s^{2} a_{5}+s a_{9}+s\right) d\left(g\left(\omega, \zeta^{*}(\omega)\right), g\left(\omega, \zeta_{n+1}(\omega)\right)\right) \\
& +\left(s^{2} a_{6}+s a_{10}\right) d\left(g\left(\omega, \xi^{*}(\omega)\right), g\left(\omega, \xi \xi_{n+1}(\omega)\right)\right) \\
& +\left(s^{2} a_{7}+s a_{11}\right) d\left(g\left(\omega, \rho^{*}(\omega)\right), g\left(\omega, \rho_{n+1}(\omega)\right)\right. \\
& +\left(s^{2} a_{8}+s a_{12}\right) d\left(g\left(\omega, \gamma^{*}(\omega)\right), g\left(\omega, \gamma_{n+1}(\omega)\right)\right) .
\end{aligned}
$$


Similarly,

$$
\begin{aligned}
d & \left.F\left(\omega,\left(\xi^{*}(\omega), \rho^{*}(\omega), \gamma^{*}(\omega), \zeta^{*}(\omega)\right)\right), g\left(\omega, \xi^{*}(\omega)\right)\right) \\
\preceq & \left(s a_{1}+s^{2} a_{13}\right) d\left(g\left(\omega, \xi^{*}(\omega)\right), F\left(\omega,\left(\xi^{*}(\omega), \rho^{*}(\omega), \gamma^{*}(\omega), \zeta^{*}(\omega)\right)\right)\right) \\
& +\left(s a_{2}+s^{2} a_{14}\right) d\left(g\left(\omega, \rho^{*}(\omega)\right), F\left(\omega,\left(\rho^{*}(\omega), \gamma^{*}(\omega), \zeta^{*}(\omega), \xi^{*}(\omega)\right)\right)\right) \\
& +\left(s a_{3}+s^{2} a_{15}\right) d\left(g\left(\omega, \gamma^{*}(\omega)\right), F\left(\omega,\left(\gamma^{*}(\omega), \zeta^{*}(\omega), \xi^{*}(\omega), \rho^{*}(\omega)\right)\right)\right) \\
& +\left(s a_{4}+s^{2} a_{16}\right) d\left(g\left(\omega, \zeta^{*}(\omega)\right), F\left(\omega,\left(\zeta^{*}(\omega), \xi^{*}(\omega), \rho^{*}(\omega), \gamma^{*}(\omega)\right)\right)\right) \\
& +\left(s^{2} a_{5}+s^{2} a_{13}+s a_{17}\right) d\left(g\left(\omega, \xi^{*}(\omega)\right), g\left(\omega, \xi_{n}(\omega)\right)\right) \\
& +\left(s^{2} a_{6}+s^{2} a_{14}+s a_{18}\right) d\left(g\left(\omega, \rho^{*}(\omega)\right), g\left(\omega, \rho_{n}(\omega)\right)\right) \\
& +\left(s^{2} a_{7}+s^{2} a_{15}+s a_{19}\right) d\left(g\left(\omega, \gamma^{*}(\omega)\right), g\left(\omega, \gamma_{n}(\omega)\right)\right) \\
& +\left(s^{2} a_{8}+s^{2} a_{16}+s a_{20}\right) d\left(g\left(\omega, \zeta^{*}(\omega)\right), g\left(\omega, \zeta_{n}(\omega)\right)\right) \\
& +\left(s^{2} a_{5}+s a_{9}+s\right) d\left(g\left(\omega, \xi^{*}(\omega)\right), g\left(\omega, \xi_{n+1}(\omega)\right)\right) \\
& +\left(s^{2} a_{6}+s a_{10}\right) d\left(g\left(\omega, \rho^{*}(\omega)\right), g\left(\omega, \rho_{n+1}(\omega)\right)\right. \\
& +\left(s^{2} a_{7}+s a_{11}\right) d\left(g\left(\omega, \gamma^{*}(\omega)\right), g\left(\omega, \gamma_{n+1}(\omega)\right)\right) \\
& +\left(s^{2} a_{8}+s a_{12}\right) d\left(g\left(\omega, \zeta^{*}(\omega)\right), g\left(\omega, \zeta_{n+1}(\omega)\right)\right),
\end{aligned}
$$

and

$$
\begin{aligned}
d & \left.F\left(\omega,\left(\rho^{*}(\omega), \gamma^{*}(\omega), \zeta^{*}(\omega), \xi^{*}(\omega)\right)\right), g\left(\omega, \rho^{*}(\omega)\right)\right) \\
\preceq & \left(s a_{1}+s^{2} a_{13}\right) d\left(g\left(\omega, \rho^{*}(\omega)\right), F\left(\omega,\left(\rho^{*}(\omega), \gamma^{*}(\omega), \zeta^{*}(\omega), \xi^{*}(\omega)\right)\right)\right) \\
& +\left(s a_{2}+s^{2} a_{14}\right) d\left(g\left(\omega, \gamma^{*}(\omega)\right), F\left(\omega,\left(\gamma^{*}(\omega), \zeta^{*}(\omega), \xi^{*}(\omega), \rho^{*}(\omega)\right)\right)\right) \\
& +\left(s a_{3}+s^{2} a_{15}\right) d\left(g\left(\omega, \zeta^{*}(\omega)\right), F\left(\omega,\left(\zeta^{*}(\omega), \xi^{*}(\omega), \rho^{*}(\omega), \gamma^{*}(\omega)\right)\right)\right) \\
& +\left(s a_{4}+s^{2} a_{16}\right) d\left(g\left(\omega, \xi^{*}(\omega)\right), F\left(\omega,\left(\xi^{*}(\omega), \rho^{*}(\omega), \gamma^{*}(\omega), \zeta^{*}(\omega)\right)\right)\right) \\
& +\left(s^{2} a_{5}+s^{2} a_{13}+s a_{17}\right) d\left(g\left(\omega, \rho^{*}(\omega)\right), g\left(\omega, \rho_{n}(\omega)\right)\right) \\
& +\left(s^{2} a_{6}+s^{2} a_{14}+s a_{18}\right) d\left(g\left(\omega, \gamma^{*}(\omega)\right), g\left(\omega, \gamma_{n}(\omega)\right)\right) \\
& +\left(s^{2} a_{7}+s^{2} a_{15}+s a_{19}\right) d\left(g\left(\omega, \zeta^{*}(\omega)\right), g\left(\omega, \zeta_{n}(\omega)\right)\right) \\
& +\left(s^{2} a_{8}+s^{2} a_{16}+s a_{20}\right) d\left(g\left(\omega, \xi^{*}(\omega)\right), g\left(\omega, \xi_{n}(\omega)\right)\right) \\
& +\left(s^{2} a_{5}+s a_{9}+s\right) d\left(g\left(\omega, \rho^{*}(\omega)\right), g\left(\omega, \rho_{n+1}(\omega)\right)\right) \\
& +\left(s^{2} a_{6}+s a_{10}\right) d\left(g\left(\omega, \gamma^{*}(\omega)\right), g(\omega, \gamma n+1(\omega))\right) \\
& +\left(s^{2} a_{7}+s a_{11}\right) d\left(g\left(\omega, \zeta^{*}(\omega)\right), g\left(\omega, \zeta_{n+1}(\omega)\right)\right) \\
& +\left(s^{2} a_{8}+s a_{12}\right) d\left(g\left(\omega, \xi^{*}(\omega)\right), g\left(\omega, \xi_{n+1}(\omega)\right)\right) .
\end{aligned}
$$

Put

$$
\begin{aligned}
\delta= & d\left(F\left(\omega,\left(\gamma^{*}(\omega), \zeta^{*}(\omega), \xi^{*}(\omega), \rho^{*}(\omega)\right)\right), g\left(\omega, \gamma^{*}(\omega)\right)\right) \\
& +d\left(F\left(\omega,\left(\gamma^{*}(\omega), \zeta^{*}(\omega), \xi^{*}(\omega), \rho^{*}(\omega)\right)\right), g\left(\omega, \gamma^{*}(\omega)\right)\right) \\
& +d\left(F\left(\omega,\left(\xi^{*}(\omega), \rho^{*}(\omega), \gamma^{*}(\omega), \zeta^{*}(\omega)\right)\right), g\left(\omega, \xi^{*}(\omega)\right)\right) \\
& +d\left(F\left(\omega,\left(\rho^{*}(\omega), \gamma^{*}(\omega), \zeta^{*}(\omega), \xi^{*}(\omega)\right)\right), g\left(\omega, \rho^{*}(\omega)\right)\right) .
\end{aligned}
$$

On view of (3.16)-(3.19), we get

$$
\begin{aligned}
\delta \preceq & \left(s a_{1}+s a_{2}+s a_{3}+s a_{4}+s^{2} a_{13}+s^{2} a_{14}+s^{2} a_{15}+s^{2} a_{16}\right) \delta \\
& +\left(s^{2} a_{5}+s^{2} a_{6}+s^{2} a_{7}+s^{2} a_{8}+s^{2} a_{13}+s^{2} a_{14}+s^{2} a_{15}+s^{2} a_{16}+s a_{17}+s a_{18}+s a_{19}+s a_{20}\right) \\
& \times d\left(g\left(\omega, \gamma^{*}(\omega)\right), g\left(\omega, \gamma_{n}(\omega)\right)\right) \\
& +\left(s^{2} a_{5}+s^{2} a_{6}+s^{2} a_{7}+s^{2} a_{8}+s^{2} a_{13}+s^{2} a_{14}+s^{2} a_{15}+s^{2} a_{16}+s a_{17}+s a_{18}+s a_{19}+s a_{20}\right)
\end{aligned}
$$




$$
\begin{aligned}
& \times d\left(g\left(\omega, \zeta^{*}(\omega)\right), g\left(\omega, \zeta_{n}(\omega)\right)\right) \\
& +\left(s^{2} a_{5}+s^{2} a_{6}+s^{2} a_{7}+s^{2} a_{8}+s^{2} a_{13}+s^{2} a_{14}+s^{2} a_{15}+s^{2} a_{16}+s a_{17}+s a_{18}+s a_{19}+s a_{20}\right) \\
& \times d\left(g\left(\omega, \xi^{*}(\omega)\right), g\left(\omega, \xi_{n}(\omega)\right)\right) \\
& +\left(s^{2} a_{5}+s^{2} a_{6}+s^{2} a_{7}+s^{2} a_{8}+s^{2} a_{13}+s^{2} a_{14}+s^{2} a_{15}+s^{2} a_{16}+s a_{17}+s a_{18}+s a_{19}+s a_{20}\right) \\
& \times d\left(g\left(\omega, \rho^{*}(\omega)\right), g\left(\omega, \rho_{n}(\omega)\right)\right) \\
& +\left(s^{2} a_{5}+s^{2} a_{6}+s^{2} a_{7}+s^{2} a_{8}+s a_{9}+s a_{10}+s a_{11}+s a_{12}+s\right) d\left(g\left(\omega, \gamma^{*}(\omega)\right), g\left(\omega, \gamma_{n+1}(\omega)\right)\right) \\
& +\left(s^{2} a_{5}+s^{2} a_{6}+s^{2} a_{7}+s^{2} a_{8}+s a_{9}+s a_{10}+s a_{11}+s a_{12}+s\right) d\left(g\left(\omega, \zeta^{*}(\omega)\right), g\left(\omega, \zeta_{n+1}(\omega)\right)\right) \\
& +\left(s^{2} a_{5}+s^{2} a_{6}+s^{2} a_{7}+s^{2} a_{8}+s a_{9}+s a_{10}+s a_{11}+s a_{12}+s\right) d\left(g\left(\omega, \xi^{*}(\omega)\right), g\left(\omega, \xi_{n+1}(\omega)\right)\right) \\
& +\left(s^{2} a_{5}+s^{2} a_{6}+s^{2} a_{7}+s^{2} a_{8}+s a_{9}+s a_{10}+s a_{11}+s a_{12}+s\right) d\left(g\left(\omega, \rho^{*}(\omega)\right), g\left(\omega, \rho_{n+1}(\omega)\right)\right) .
\end{aligned}
$$

Then

$$
\begin{aligned}
\delta \preceq & \frac{B}{e-A} d\left(g\left(\omega, \gamma^{*}(\omega)\right), g\left(\omega, \gamma_{n}(\omega)\right)\right)+\frac{B}{e-A} d\left(g\left(\omega, \zeta^{*}(\omega)\right), g\left(\omega, \zeta_{n}(\omega)\right)\right) \\
& +\frac{B}{e-A} d\left(g\left(\omega, \xi^{*}(\omega)\right), g\left(\omega, \xi_{n}(\omega)\right)\right)+\frac{B}{e-A} d\left(g\left(\omega, \rho^{*}(\omega)\right), g\left(\omega, \rho_{n}(\omega)\right)\right) \\
& +\frac{C}{e-A} d\left(g\left(\omega, \gamma^{*}(\omega)\right), g\left(\omega, \gamma_{n+1}(\omega)\right)+\frac{C}{e-A} d\left(g\left(\omega, \zeta^{*}(\omega)\right), g\left(\omega, \zeta_{n+1}(\omega)\right)\right)\right. \\
& +\frac{C}{e-A} d\left(g\left(\omega, \xi^{*}(\omega)\right), g\left(\omega, \xi_{n+1}(\omega)\right)\right)+\frac{C}{e-A} d\left(g\left(\omega, \rho^{*}(\omega)\right), g\left(\omega, \rho_{n+1}(\omega)\right)\right),
\end{aligned}
$$

where $A=s a_{1}+s a_{2}+s a_{3}+s a_{4}+s^{2} a_{13}+s^{2} a_{14}+s^{2} a_{15}+s^{2} a_{16}, B=s^{2} a_{5}+s^{2} a_{6}+s^{2} a_{7}+s^{2} a_{8}+s^{2} a_{13}+$ $s^{2} a_{14}+s^{2} a_{15}+s^{2} a_{16}+s a_{17}+s a_{18}+s a_{19}+s a_{20}, C=s^{2} a_{5}+s^{2} a_{6}+s^{2} a_{7}+s^{2} a_{8}+s a_{9}+s a_{10}+s a_{11}+$ $s a_{12}+s, \rho(A)<1$. Since $g\left(\omega,\left(\gamma_{n}(\omega)\right) \rightarrow g\left(\omega,\left(\gamma^{*}(\omega)\right), g\left(\omega,\left(\zeta_{n}(\omega)\right) \rightarrow g\left(\omega,\left(\zeta^{*}(\omega)\right), g\left(\omega,\left(\xi_{n}(\omega)\right) \rightarrow\right.\right.\right.\right.\right.$ $g\left(\omega,\left(\xi^{*}(\omega)\right), g\left(\omega,\left(\rho_{n}(\omega)\right) \rightarrow g\left(\omega,\left(\rho^{*}(\omega)\right)\right.\right.\right.$, then Lemma 2.7 follows that for any $c \gg \theta$, there exists $N_{0}$ such that for $n>N_{0}$, we have

$$
\begin{aligned}
\frac{B}{e-A} \mathrm{~d}\left(g\left(\omega, \gamma^{*}(\omega)\right), g\left(\omega, \gamma_{n}(\omega)\right)\right) & \ll \frac{c}{8}, & \frac{B}{e-A} \mathrm{~d}\left(g\left(\omega, \zeta^{*}(\omega)\right), g\left(\omega, \zeta_{n}(\omega)\right)\right) & \ll \frac{c}{8}, \\
\frac{B}{e-A} \mathrm{~d}\left(g\left(\omega, \xi^{*}(\omega)\right), g\left(\omega, \xi_{n}(\omega)\right)\right) & \ll \frac{c}{8}, & \frac{B}{e-A} \mathrm{~d}\left(g\left(\omega, \rho^{*}(\omega)\right), g\left(\omega, \rho_{n}(\omega)\right)\right) & \ll \frac{c}{8}, \\
\frac{C}{e-A} \mathrm{~d}\left(g\left(\omega, \gamma^{*}(\omega)\right), g\left(\omega, \gamma_{n+1}(\omega)\right)\right) & \ll \frac{c}{8}, & \frac{C}{e-A} \mathrm{~d}\left(g\left(\omega, \zeta^{*}(\omega)\right), g\left(\omega, \zeta_{n+1}(\omega)\right)\right) & \ll \frac{c}{8}, \\
\frac{C}{e-A} \mathrm{~d}\left(g\left(\omega, \xi^{*}(\omega)\right), g\left(\omega, \xi_{n+1}(\omega)\right)\right) & \ll \frac{c}{8}, & \frac{C}{e-A} \mathrm{~d}\left(g\left(\omega, \rho^{*}(\omega)\right), g\left(\omega, \rho_{n+1}(\omega)\right)\right) & \ll \frac{c}{8} .
\end{aligned}
$$

Hence,

$$
\delta \ll \frac{c}{8}+\frac{c}{8}+\frac{c}{8}+\frac{c}{8}+\frac{c}{8}+\frac{c}{8}+\frac{c}{8}+\frac{c}{8}=c .
$$

Now, according to Lemma 2.4, it follows that $\delta=\theta$, that is,

$$
\begin{aligned}
\theta= & d\left(F\left(\omega,\left(\gamma^{*}(\omega), \zeta^{*}(\omega), \xi^{*}(\omega), \rho^{*}(\omega)\right)\right), g\left(\omega, \gamma^{*}(\omega)\right)\right) \\
& +d\left(F\left(\omega,\left(\gamma^{*}(\omega), \zeta^{*}(\omega), \xi^{*}(\omega), \rho^{*}(\omega)\right)\right), g\left(\omega, \gamma^{*}(\omega)\right)\right) \\
& +d\left(F\left(\omega,\left(\xi^{*}(\omega), \rho^{*}(\omega), \gamma^{*}(\omega), \zeta^{*}(\omega)\right)\right), g\left(\omega, \xi^{*}(\omega)\right)\right) \\
& +d\left(F\left(\omega,\left(\rho^{*}(\omega), \gamma^{*}(\omega), \zeta^{*}(\omega), \xi^{*}(\omega)\right)\right), g\left(\omega, \rho^{*}(\omega)\right)\right),
\end{aligned}
$$

which implies that

$$
\begin{aligned}
& d\left(F\left(\omega,\left(\gamma^{*}(\omega), \zeta^{*}(\omega), \xi^{*}(\omega), \rho^{*}(\omega)\right)\right), g\left(\omega, \gamma^{*}(\omega)\right)\right)=\theta, \\
& d\left(F\left(\omega,\left(\gamma^{*}(\omega), \zeta^{*}(\omega), \xi^{*}(\omega), \rho^{*}(\omega)\right)\right), g\left(\omega, \gamma^{*}(\omega)\right)\right)=\theta,
\end{aligned}
$$




$$
\begin{aligned}
& d\left(F\left(\omega,\left(\xi^{*}(\omega), \rho^{*}(\omega), \gamma^{*}(\omega), \zeta^{*}(\omega)\right)\right), g\left(\omega, \xi^{*}(\omega)\right)\right)=\theta \\
& d\left(F\left(\omega,\left(\rho^{*}(\omega), \gamma^{*}(\omega), \zeta^{*}(\omega), \xi^{*}(\omega)\right)\right), g\left(\omega, \rho^{*}(\omega)\right)\right)=\theta .
\end{aligned}
$$

Thus,

$$
\begin{aligned}
& d\left(F\left(\omega,\left(\gamma^{*}(\omega), \zeta^{*}(\omega), \zeta^{*}(\omega), \rho^{*}(\omega)\right)\right)\right)=g\left(\omega, \gamma^{*}(\omega)\right), \\
& d\left(F\left(\omega,\left(\gamma^{*}(\omega), \zeta^{*}(\omega), \xi^{*}(\omega), \rho^{*}(\omega)\right)\right)\right)=g\left(\omega, \zeta^{*}(\omega)\right), \\
& d\left(F\left(\omega,\left(\xi^{*}(\omega), \rho^{*}(\omega), \gamma^{*}(\omega), \zeta^{*}(\omega)\right)\right)\right)=g\left(\omega, \xi^{*}(\omega)\right), \\
& d\left(F\left(\omega,\left(\rho^{*}(\omega), \gamma^{*}(\omega), \zeta^{*}(\omega), \xi^{*}(\omega)\right)\right)\right)=g\left(\omega, \rho^{*}(\omega)\right) .
\end{aligned}
$$

Therefore $\left(\gamma^{*}(\omega), \zeta^{*}(\omega), \xi^{*}(\omega), \rho^{*}(\omega)\right)$ is a quadruple random coincidence point of $F$ and $g$ for all $\omega \in$ $\Omega$.

Corollary 3.2. Let $(\mathrm{X}, \mathrm{d})$ be a separable cone b-metric space over Banach algebra $\mathcal{A}$ and $\mathrm{P}$ be a cone in $\mathcal{A}, \mathrm{s} \geqslant 1$ be a constant, and $(\Omega, \Sigma)$ be a measurable space. Suppose that the mappings $\mathrm{F}: \Omega \times \mathrm{X}^{4} \rightarrow \mathrm{X}, \mathrm{g}: \Omega \times \mathrm{X} \rightarrow \mathrm{X}$ satisfy the following contractive condition:

$$
\begin{aligned}
d\left(F\left(\omega,\left(x_{1}, x_{2}, x_{3}, x_{4}\right)\right), F\left(\omega,\left(x_{1}^{*}, x_{2}^{*}, x_{3}^{*}, x_{4}^{*}\right)\right)\right) \preceq & a_{1} d\left(g\left(\omega, x_{1}\right), g\left(\omega, x_{1}^{*}\right)\right)+a_{2} d\left(g\left(\omega, x_{2}\right), g\left(\omega, x_{2}^{*}\right)\right) \\
& +a_{3} d\left(g\left(\omega, x_{3}\right), g\left(\omega, x_{3}^{*}\right)\right)+a_{3} d\left(g\left(\omega, x_{4}\right), g\left(\omega, x_{4}^{*}\right)\right)
\end{aligned}
$$

for all $x_{1}, x_{2}, x_{3}, x_{4}, x_{1}^{*}, x_{2}^{*}, x_{3}^{*}, x_{4}^{*}$, where $a_{1}, a_{2}, a_{3}, a_{4} \in P$ are generalized Lipschitz constants with $p\left(a_{1}+a_{2}+\right.$ $\left.\mathrm{a}_{3}+\mathrm{a}_{4}\right)<\frac{1}{\mathrm{~s}}, \mathrm{~F}(\cdot, v), \mathrm{g}(\cdot, \mathrm{x})$ are measurable for $v \in \mathrm{x}^{4}$ and $\mathrm{x} \in \mathrm{X}$, respectively, and $\mathrm{F}\left(\omega \times \mathrm{X}^{4}\right) \subseteq \mathrm{g}(\omega \times \mathrm{X})$ is complete subspace of $X$ for each $\omega \in \Omega$, then there are mappings $\gamma, \zeta, \xi, \rho: \Omega \rightarrow X$ such that

$$
\begin{aligned}
& F(\omega,(\gamma(\omega), \zeta(\omega), \xi(\omega), \rho(\omega)))=g(\omega, \gamma(\omega)), \\
& F(\omega,(\zeta(\omega), \xi(\omega), \rho(\omega), \gamma(\omega)))=g(\omega, \zeta(\omega)), \\
& F(\omega,(\xi(\omega), \rho(\omega), \gamma(\omega), \zeta(\omega)))=g(\omega, \xi(\omega)), \\
& F(\omega,(\rho(\omega), \gamma(\omega), \zeta(\omega), \xi(\omega)))=g(\omega, \rho(\omega))
\end{aligned}
$$

for all $\omega \in \Omega$, that is $\mathrm{F}$ and $\mathrm{g}$ have a quadruple random coincidence point.

Theorem 3.3. In addition to hypotheses of Theorem 3.1, if $\mathrm{F}$ and $\mathrm{g}$ are $w$-compatible, then $\mathrm{F}$ and $\mathrm{g}$ have a unique quadruple common fixed point. Moreover, a quadruple random common fixed point of $\mathrm{F}$ and $\mathrm{g}$ is of the form $\left(\gamma^{*}(\omega), \gamma^{*}(\omega), \gamma^{*}(\omega), \gamma^{*}(\omega)\right) \in \mathrm{X}$ for all $\omega \in \Omega$.

Proof. By Theorem 3.1, F and $g$ have quadruple random coincidence point $\left(\gamma^{*}(\omega), \zeta^{*}(\omega), \xi^{*}(\omega), \rho^{*}(\omega)\right)$. Then $\left(g\left(\omega, \gamma^{*}(\omega)\right), g\left(\omega, \zeta^{*}(\omega)\right), g\left(\omega, \xi^{*}(\omega)\right), g\left(\omega, \rho^{*}(\omega)\right)\right)$ is quadruple random point of coincidence of $F$ and $g$ such that

$$
\begin{aligned}
& g\left(\omega, \gamma^{*}(\omega)\right)=F\left(\omega,\left(\gamma^{*}(\omega), \zeta^{*}(\omega), \xi^{*}(\omega), \rho^{*}(\omega)\right)\right), \\
& g\left(\omega, \zeta^{*}(\omega)\right)=F\left(\omega,\left(\zeta^{*}(\omega), \xi^{*}(\omega), \rho^{*}(\omega), \gamma^{*}(\omega)\right)\right), \\
& g\left(\omega, \xi^{*}(\omega)\right)=F\left(\omega,\left(\xi^{*}(\omega), \rho^{*}(\omega), \gamma^{*}(\omega), \zeta^{*}(\omega)\right)\right), \\
& g\left(\omega, \rho^{*}(\omega)\right)=F\left(\omega,\left(\rho^{*}(\omega), \gamma^{*}(\omega), \zeta^{*}(\omega), \xi^{*}(\omega)\right)\right) .
\end{aligned}
$$

First, we shall show that the quadruple random point of coincidence is unique. Suppose that $F$ and $g$ have another quadruple random point of coincidence

$$
\left(g\left(\omega, \gamma^{* *}(\omega)\right), g\left(\omega, \zeta^{* *}(\omega)\right), g\left(\omega, \xi^{* *}(\omega)\right), g\left(\omega, \rho^{* *}(\omega)\right)\right),
$$

such that

$$
g\left(\omega, \gamma^{* *}(\omega)\right)=F\left(\omega,\left(\gamma^{* *}(\omega), \zeta^{* *}(\omega), \xi^{* *}(\omega), \rho^{* *}(\omega)\right)\right)
$$




$$
\begin{aligned}
& g\left(\omega, \zeta^{* *}(\omega)\right)=F\left(\omega,\left(\zeta^{* *}(\omega), \xi^{* *}(\omega), \rho^{* *}(\omega), \gamma^{* *}(\omega)\right)\right), \\
& g\left(\omega, \xi^{* *}(\omega)\right)=F\left(\omega,\left(\xi^{* *}(\omega), \rho^{* *}(\omega), \gamma^{* *}(\omega), \zeta^{* *}(\omega)\right)\right), \\
& g\left(\omega, \rho^{* *}(\omega)\right)=F\left(\omega,\left(\rho^{* *}(\omega), \gamma^{* *}(\omega), \zeta^{* *}(\omega), \xi^{* *}(\omega)\right)\right),
\end{aligned}
$$

where $\left(\gamma^{* *}(\omega), \zeta^{* *}(\omega), \xi^{* *}(\omega), \rho^{* *}(\omega)\right) \in X^{4}$ for all $\omega \in \Omega$. Then we have

$$
\begin{aligned}
& \mathrm{d}\left(\mathrm{g}\left(\omega, \gamma^{*}(\omega)\right), \mathrm{g}\left(\omega, \gamma^{* *}(\omega)\right)\right) \\
& =d\left(F\left(\omega,\left(\gamma^{*}(\omega), \zeta^{*}(\omega), \xi^{*}(\omega), \rho^{*}(\omega)\right)\right), F\left(\omega,\left(\gamma^{* *}(\omega), \zeta^{* *}(\omega), \xi^{* *}(\omega), \rho^{* *}(\omega)\right)\right)\right) \\
& \preceq\left[a _ { 1 } d \left(g\left(\omega, \gamma^{*}(\omega)\right), F\left(\omega,\left(\gamma^{*}(\omega), \zeta^{*}(\omega), \xi^{*}(\omega), \rho^{*}(\omega)\right)\right)\right.\right. \\
& +a_{2} d\left(g\left(\omega, \zeta^{*}(\omega)\right), F\left(\omega,\left(\zeta^{*}(\omega), \xi^{*}(\omega), \rho^{*}(\omega), \gamma^{*}(\omega)\right)\right)\right. \\
& +a_{3} d\left(g\left(\omega, \xi^{*}(\omega)\right), F\left(\omega,\left(\xi^{*}(\omega), \rho^{*}(\omega), \gamma^{*}(\omega), \zeta^{*}(\omega)\right)\right)\right. \\
& +a_{4} d\left(g\left(\omega, \rho^{*}(\omega)\right), F\left(\omega,\left(\rho^{*}(\omega), \gamma^{*}(\omega), \zeta^{*}(\omega), \xi^{*}(\omega)\right)\right)\right] \\
& +\left[a _ { 5 } d \left(g\left(\omega, \gamma^{* *}(\omega)\right), F\left(\omega,\left(\gamma^{* *}(\omega), \zeta^{* *}(\omega), \xi^{* *}(\omega), \rho^{* *}(\omega)\right)\right)\right.\right. \\
& +a_{6} d\left(g\left(\omega, \zeta^{* *}(\omega)\right), F\left(\omega,\left(\zeta^{* *}(\omega), \xi^{* *}(\omega), \rho^{* *}(\omega), \gamma^{* *}(\omega)\right)\right)\right. \\
& +a_{7} d\left(g\left(\omega, \xi^{* *}(\omega)\right), F\left(\omega,\left(\xi^{* *}(\omega), \rho^{* *}(\omega), \gamma^{* *}(\omega), \zeta^{* *}(\omega)\right)\right)\right. \\
& +a_{8} d\left(g\left(\omega, \rho^{* *}(\omega)\right), F\left(\omega,\left(\rho^{* *}(\omega), \gamma^{* *}(\omega), \zeta^{* *}(\omega), \xi^{* *}(\omega)\right)\right)\right] \\
& +\left[a _ { 9 } d \left(g\left(\omega, \gamma^{*}(\omega)\right), F\left(\omega,\left(\gamma^{* *}(\omega), \zeta^{* *}(\omega), \xi^{* *}(\omega), \rho^{* *}(\omega)\right)\right)\right.\right. \\
& +a_{10} d\left(g\left(\omega, \zeta^{*}(\omega)\right), F\left(\omega,\left(\zeta^{* *}(\omega), \xi^{* *}(\omega), \rho^{* *}(\omega), \gamma^{* *}(\omega)\right)\right)\right. \\
& +a_{11} d\left(g\left(\omega, \xi^{*}(\omega)\right), F\left(\omega,\left(\xi^{* *}(\omega), \rho^{* *}(\omega), \gamma^{* *}(\omega), \zeta^{* *}(\omega)\right)\right)\right. \\
& +a_{12} d\left(g\left(\omega, \rho^{*}(\omega)\right), F\left(\omega,\left(\rho^{* *}(\omega), \gamma^{* *}(\omega), \zeta^{* *}(\omega), \xi^{* *}(\omega)\right)\right)\right] \\
& +\left[a _ { 1 3 } d \left(g\left(\omega, \gamma^{* *}(\omega)\right), F\left(\omega,\left(\gamma^{*}(\omega), \zeta^{*}(\omega), \xi^{*}(\omega), \rho^{*}(\omega)\right)\right)\right.\right. \\
& +a_{14} d\left(g\left(\omega, \zeta^{* *}(\omega)\right), F\left(\omega,\left(\zeta^{*}(\omega), \zeta^{*}(\omega), \rho^{*}(\omega), \gamma^{*}(\omega)\right)\right)\right. \\
& +a_{15} d\left(g\left(\omega, \xi^{* *}(\omega)\right), F\left(\omega, \xi^{*}(\omega), \rho^{*}(\omega), \gamma^{*}(\omega), \zeta^{*}(\omega)\right)\right) \\
& +a_{16} d\left(g\left(\omega, \rho^{* *}(\omega)\right), F\left(\omega,\left(\rho^{*}(\omega), \gamma^{*}(\omega), \zeta^{*}(\omega), \xi^{*}(\omega)\right)\right)\right] \\
& +\left[a_{17} d\left(g\left(\omega, \gamma^{*}(\omega)\right), g\left(\omega, \gamma^{* *}(\omega)\right)\right)+a_{18} d\left(g\left(\omega, \zeta^{*}(\omega)\right), g\left(\omega, \zeta^{* *}(\omega)\right)\right)\right. \\
& \left.+a_{19} d\left(g\left(\omega, \xi^{*}(\omega)\right), g\left(\omega, \xi^{* *}(\omega)\right)\right)+a_{20} d\left(g\left(\omega, \rho^{*}(\omega)\right), g\left(\omega, \rho^{* *}(\omega)\right)\right)\right] \\
& =\left[a_{1} d\left(g\left(\omega, \gamma^{*}(\omega)\right), g\left(\omega, \gamma^{*}(\omega)\right)\right)+a_{2} d\left(g\left(\omega, \zeta^{*}(\omega)\right), g\left(\omega, \zeta^{*}(\omega)\right)\right)\right. \\
& +a_{3} d\left(g\left(\omega, \xi^{*}(\omega)\right), g\left(\omega, \xi^{*}(\omega)\right)+a_{4} d\left(g\left(\omega, \rho^{*}(\omega)\right), g\left(\omega, \rho^{*}(\omega)\right)\right)\right] \\
& +\left[a_{5} d\left(g\left(\omega, \gamma^{* *}(\omega)\right), g\left(\omega, \gamma^{* *}(\omega)\right)\right)+a_{6} d\left(g\left(\omega, \zeta^{* *}(\omega)\right), g\left(\omega, \zeta^{* *}(\omega)\right)\right)\right. \\
& \left.+a_{7} d\left(g\left(\omega, \xi^{* *}(\omega)\right), g\left(\omega, \xi^{* *}(\omega)\right)\right)+a_{8} d\left(g\left(\omega, \rho^{* *}(\omega)\right), g\left(\omega, \rho^{* *}(\omega)\right)\right)\right] \\
& +\left[a_{9} d\left(g\left(\omega, \gamma^{*}(\omega)\right), g\left(\omega, \gamma^{* *}(\omega)\right)\right)+a_{10} d\left(g\left(\omega, \zeta^{*}(\omega)\right), g\left(\omega, \zeta^{* *}(\omega)\right)\right)\right. \\
& \left.+a_{11} d\left(g\left(\omega, \xi^{*}(\omega)\right), g\left(\omega, \xi^{* *}(\omega)\right)\right)+a_{12} d\left(g\left(\omega, \rho^{*}(\omega)\right), g\left(\omega, \rho^{* *}(\omega)\right)\right)\right] \\
& +\left[a_{13} d\left(g\left(\omega, \gamma^{* *}(\omega)\right), g\left(\omega, \gamma^{*}(\omega)\right)\right)+a_{14} d\left(g\left(\omega, \zeta^{* *}(\omega)\right), g\left(\omega, \zeta^{*}(\omega)\right)\right)\right. \\
& \left.+a_{15} d\left(g\left(\omega, \xi^{* *}(\omega)\right), g\left(\omega, \xi^{*}(\omega)\right)\right)+a_{16} d\left(g\left(\omega, \rho^{* *}(\omega)\right), g\left(\omega, \rho^{*}(\omega)\right)\right)\right] \\
& +\left[a_{17} d\left(g\left(\omega, \gamma^{*}(\omega)\right), g\left(\omega, \gamma^{* *}(\omega)\right)\right)+a_{18} d\left(g\left(\omega, \zeta^{*}(\omega)\right), g\left(\omega, \zeta^{* *}(\omega)\right)\right)\right. \\
& \left.+a_{19} d\left(g\left(\omega, \xi^{*}(\omega)\right), g\left(\omega, \xi^{* *}(\omega)\right)\right)+a_{20} d\left(g\left(\omega, \rho^{*}(\omega)\right), g\left(\omega, \rho^{* *}(\omega)\right)\right)\right] \text {. }
\end{aligned}
$$

Hence,

$$
\begin{aligned}
d\left(g\left(\omega, \gamma^{*}(\omega)\right), g\left(\omega, \gamma^{* *}(\omega)\right)\right) \preceq & \left(a_{9}+a_{13}+a_{17}\right) d\left(g\left(\omega, \gamma^{*}(\omega)\right), g\left(\omega, \gamma^{* *}(\omega)\right)\right) \\
& +\left(a_{10}+a_{14}+a_{18}\right) d\left(g\left(\omega, \zeta^{*}(\omega)\right), g\left(\omega, \zeta^{* *}(\omega)\right)\right) \\
& +\left(a_{11}+a_{15}+a_{19}\right) d\left(g\left(\omega, \xi^{*}(\omega)\right), g\left(\omega, \xi^{* *}(\omega)\right)\right) \\
& +\left(a_{12}+a_{16}+a_{20}\right) d\left(g\left(\omega, \rho^{*}(\omega)\right), g\left(\omega, \rho^{* *}(\omega)\right)\right) .
\end{aligned}
$$


Similarly, we can prove that

$$
\begin{aligned}
d\left(g\left(\omega, \zeta^{*}(\omega)\right), g\left(\omega, \zeta^{* *}(\omega)\right)\right) \preceq & \left(a_{9}+a_{13}+a_{17}\right) d\left(g\left(\omega, \zeta^{*}(\omega)\right), g\left(\omega, \zeta^{* *}(\omega)\right)\right) \\
& +\left(a_{10}+a_{14}+a_{18}\right) d\left(g\left(\omega, \xi^{*}(\omega)\right), g\left(\omega, \xi^{* *}(\omega)\right)\right) \\
& +\left(a_{11}+a_{15}+a_{19}\right) d\left(g\left(\omega, \rho^{*}(\omega)\right), g\left(\omega, \rho^{* *}(\omega)\right)\right) \\
& +\left(a_{12}+a_{16}+a_{20}\right) d\left(g\left(\omega, \gamma^{*}(\omega)\right), g\left(\omega, \gamma^{* *}(\omega)\right)\right),
\end{aligned}
$$

and

$$
\begin{aligned}
d\left(g\left(\omega, \xi^{*}(\omega)\right), g\left(\omega, \xi^{* *}(\omega)\right)\right) \preceq & \left(a_{9}+a_{13}+a_{17}\right) d\left(g\left(\omega, \xi^{*}(\omega)\right), g\left(\omega, \xi^{* *}(\omega)\right)\right) \\
& +\left(a_{10}+a_{14}+a_{18}\right) d\left(g\left(\omega, \rho^{*}(\omega)\right), g\left(\omega, \rho^{* *}(\omega)\right)\right) \\
& +\left(a_{11}+a_{15}+a_{19}\right) d\left(g\left(\omega, \gamma^{*}(\omega)\right), g\left(\omega, \gamma^{* *}(\omega)\right)\right) \\
& +\left(a_{12}+a_{16}+a_{20}\right) d\left(g\left(\omega, \zeta^{*}(\omega)\right), g\left(\omega, \zeta^{* *}(\omega)\right)\right),
\end{aligned}
$$

and

$$
\begin{aligned}
d\left(g\left(\omega, \rho^{*}(\omega)\right), g\left(\omega, \rho^{* *}(\omega)\right)\right) \preceq & \left(a_{9}+a_{13}+a_{17}\right) d\left(g\left(\omega, \rho^{*}(\omega)\right), g\left(\omega, \rho^{* *}(\omega)\right)\right) \\
& +\left(a_{10}+a_{14}+a_{18}\right) d\left(g\left(\omega, \gamma^{*}(\omega)\right), g\left(\omega, \gamma^{* *}(\omega)\right)\right) \\
& +\left(a_{11}+a_{15}+a_{19}\right) d\left(g\left(\omega, \zeta^{*}(\omega)\right), g\left(\omega, \zeta^{* *}(\omega)\right)\right) \\
& +\left(a_{12}+a_{16}+a_{20}\right) d\left(g\left(\omega, \xi^{*}(\omega)\right), g\left(\omega, \xi^{* *}(\omega)\right)\right) .
\end{aligned}
$$

By combining (3.20)-(3.23), we get

$$
\begin{aligned}
& d\left(g\left(\omega, \gamma^{*}(\omega)\right), g\left(\omega, \gamma^{* *}(\omega)\right)\right)+d\left(g\left(\omega, \zeta^{*}(\omega)\right), g\left(\omega, \zeta^{* *}(\omega)\right)\right) \\
& \quad+d\left(g\left(\omega, \xi^{*}(\omega)\right), g\left(\omega, \xi^{* *}(\omega)\right)\right)+d\left(g\left(\omega, \rho^{*}(\omega)\right), g\left(\omega, \rho^{* *}(\omega)\right)\right) \\
& \preceq\left(a_{9}+a_{10}+a_{11}+a_{12}+a_{13}+a_{14}+a_{15}+a_{16}+a_{17}+a_{18}+a_{19}+a_{20}\right)\left(d\left(g\left(\omega, \gamma^{*}(\omega)\right), g\left(\omega, \gamma^{* *}(\omega)\right)\right)\right. \\
&\left.\quad+d\left(g\left(\omega, \zeta^{*}(\omega)\right), g\left(\omega, \zeta^{* *}(\omega)\right)\right)+d\left(g\left(\omega, \xi^{*}(\omega)\right), g\left(\omega, \xi^{* *}(\omega)\right)\right)+d\left(g\left(\omega, \rho^{*}(\omega)\right), g\left(\omega, \rho^{* *}(\omega)\right)\right)\right) .
\end{aligned}
$$

Set $\lambda=a_{9}+a_{10}+a_{11}+a_{12}+a_{13}+a_{14}+a_{15}+a_{16}+a_{17}+a_{18}+a_{19}+a_{20}$, and

$$
\begin{aligned}
\beta= & d\left(g\left(\omega, \gamma^{*}(\omega)\right), g\left(\omega, \gamma^{* *}(\omega)\right)\right)+d\left(g\left(\omega, \zeta^{*}(\omega)\right), g\left(\omega, \zeta^{* *}(\omega)\right)\right) \\
& +d\left(g\left(\omega, \xi^{*}(\omega)\right), g\left(\omega, \xi^{* *}(\omega)\right)\right)+d\left(g\left(\omega, \rho^{*}(\omega)\right), g\left(\omega, \rho^{* *}(\omega)\right)\right) .
\end{aligned}
$$

We have $\beta \preceq \lambda \beta \preceq \cdots \preceq \lambda^{n} \beta$. Now we get that

$$
\rho(\lambda) \leqslant \rho\left(a_{9}+\cdots+a_{16}\right)+\rho\left(a_{17}+a_{18}+a_{19}+a_{20}\right)<1,
$$

which leads to $\lambda^{n} \rightarrow \theta\left(n \rightarrow \infty\right.$, we claim that, for each $c \gg \theta$, there exists $n_{0}(c)$ such that $\lambda^{n} \ll c\left(n>n_{0}(c)\right)$. Consequently by Lemma 2.6,

$$
\begin{aligned}
& d\left(g\left(\omega, \gamma^{*}(\omega)\right), g\left(\omega, \gamma^{* *}(\omega)\right)\right)+d\left(g\left(\omega, \zeta^{*}(\omega)\right), g\left(\omega, \zeta^{* *}(\omega)\right)\right) \\
& \quad+d\left(g\left(\omega, \xi^{*}(\omega)\right), g\left(\omega, \xi^{* *}(\omega)\right)\right)+d\left(g\left(\omega, \rho^{*}(\omega)\right), g\left(\omega, \rho^{* *}(\omega)\right)\right)=\theta .
\end{aligned}
$$

Hence,

$$
\begin{array}{ll}
d\left(g\left(\omega, \gamma^{*}(\omega)\right), g\left(\omega, \gamma^{* *}(\omega)\right)\right)=\theta, & d\left(g\left(\omega, \zeta^{*}(\omega)\right), g\left(\omega, \zeta^{* *}(\omega)\right)\right)=\theta, \\
d\left(g\left(\omega, \xi^{*}(\omega)\right), g\left(\omega, \xi^{* *}(\omega)\right)\right)=\theta, & d\left(g\left(\omega, \rho^{*}(\omega)\right), g\left(\omega, \rho^{* *}(\omega)\right)\right)=\theta
\end{array}
$$

that is,

$$
\begin{array}{ll}
g\left(\omega, \gamma^{*}(\omega)\right)=g\left(\omega, \gamma^{* *}(\omega)\right), & g\left(\omega, \zeta^{*}(\omega)\right)=g\left(\omega, \zeta^{* *}(\omega)\right), \\
g\left(\omega, \xi^{*}(\omega)\right)=g\left(\omega, \xi^{* *}(\omega)\right), & g\left(\omega, \rho^{*}(\omega)\right)=g\left(\omega, \rho^{* *}(\omega)\right),
\end{array}
$$


which implies the uniqueness of the quadruple random point of coincidence of $F$ and $g$. By a similar way, someone can prove that

$$
\begin{array}{ll}
g\left(\omega, \gamma^{*}(\omega)\right)=g\left(\omega, \zeta^{* *}(\omega)\right), & g\left(\omega, \zeta^{*}(\omega)\right)=g\left(\omega, \xi^{* *}(\omega)\right), \\
g\left(\omega, \xi^{*}(\omega)\right)=g\left(\omega, \rho^{* *}(\omega)\right), & g\left(\omega, \rho^{*}(\omega)\right)=g\left(\omega, \gamma^{* *}(\omega)\right), \\
g\left(\omega, \gamma^{*}(\omega)\right)=g\left(\omega, \xi^{* *}(\omega)\right), & g\left(\omega, \zeta^{*}(\omega)\right)=g\left(\omega, \rho^{* *}(\omega)\right), \\
g\left(\omega, \xi^{*}(\omega)\right)=g\left(\omega, \gamma^{* *}(\omega)\right), & g\left(\omega, \rho^{*}(\omega)\right)=g\left(\omega, \zeta^{* *}(\omega)\right), \\
g\left(\omega, \gamma^{*}(\omega)\right)=g\left(\omega, \rho^{* *}(\omega)\right), & g\left(\omega, \zeta^{*}(\omega)\right)=g\left(\omega, \gamma^{* *}(\omega)\right) \\
g\left(\omega, \xi^{*}(\omega)\right)=g\left(\omega, \zeta^{* *}(\omega)\right), & g\left(\omega, \rho^{*}(\omega)\right)=g\left(\omega, \xi^{* *}(\omega)\right),
\end{array}
$$

In view of (3.24)-(3.27), one can assert

$$
g\left(\omega, \gamma^{*}(\omega)\right)=g\left(\omega, \zeta^{*}(\omega)\right)=g\left(\omega, \xi^{*}(\omega)\right)=g\left(\omega, \rho^{*}(\omega)\right) .
$$

In other words, the unique quadruple random point of coincidence of $F$ and $g$ is $\left(g\left(\omega, \gamma^{*}(\omega)\right), g\left(\omega, \zeta^{*}(\omega)\right)\right.$, $\left.g\left(\omega, \xi^{*}(\omega)\right), g\left(\omega, \rho^{*}(\omega)\right)\right)$. have

Let $u(\omega)=g\left(\omega, \gamma^{*}\right)=F\left(\omega,\left(\gamma^{*}(\omega), \zeta^{*}(\omega), \xi^{*}(\omega), \rho^{*}(\omega)\right)\right)$. Since $F$ and $g$ are $w$-compatible, then we

$$
\begin{aligned}
g(\omega, \mathrm{u}(\omega)) & =g\left(g\left(\omega, \gamma^{*}(\omega)\right)\right) \\
& =g\left(F\left(\omega,\left(\gamma^{*}(\omega), \zeta^{*}(\omega), \xi^{*}(\omega), \rho^{*}(\omega)\right)\right)\right) \\
& =F\left(\omega,\left(g\left(\omega, \gamma^{*}(\omega)\right), g\left(\omega, \zeta^{*}(\omega)\right), g\left(\omega, \xi^{*}(\omega)\right), g\left(\omega, \rho^{*}(\omega)\right)\right)\right) \\
& =F(\omega,(u(\omega), u(\omega), u(\omega), u(\omega))) .
\end{aligned}
$$

Hence $(u(\omega), u(\omega), u(\omega), u(\omega))$ is the unique quadruple common random fixed point of $F$ and $g$ for all $\omega \in \Omega$. This completes the proof.

\section{Acknowledgment}

This project was partially supported by Faculty of Science, KMUTT. The authors acknowledge the financial support provided by King Mongkut's University of Technology Thonburi through the "KMUTT $55^{\text {th }}$ Aniversary Commemorative Fund". Moreover, Chayut Kongban would like to thank the Petchra Pra Jom Klao Ph.D. Research Scholarship and the King Mongkut's University of Technology Thonburi (KMUTT) for financial support.

\section{References}

[1] A. Alotaibi, S. M. Alsulami, Coupled coincidence points for monotone operators in partially ordered metric spaces, Fixed Point Theory Appl., 2011 (2011), 13 pages. 1

[2] I. Altun, B. Damjanović, D. Djorić, Fixed point and common fixed point theorems on ordered cone metric spaces, Appl. Math. Lett., 23 (2010), 310-316. 1

[3] A. D. Arvanitakis, A proof of the generalized Banach contraction conjecture, Proc. Amer. Math. Soc., 131 (2003), $3647-$ 3656. 1

[4] I. A. Bakhtin, The contraction mapping principle in almost metric space, (Russian) Functional analysis, Ul'yanovsk. Gos. Ped. Inst., Ul'yanovsk, 30 (1989), 26-37. 1

[5] D. W. Boyd, J. S. W. Wong, On nonlinear contractions, Proc. Amer. Math. Soc., 20 (1969), 458-464. 1

[6] L. Ćirić, V. Lakshmikantham, Coupled random fixed point theorems for nonlinear contractions in partially ordered metric spaces, Stoch. Anal. Appl., 27 (2009), 1246-1259. 1

[7] Y. Han, S.-Y. Xu, Some new theorems of expanding mappings without continuity in cone metric spaces, Fixed point Theory Appl., 2013 (2013), 9 pages. 1

[8] C. J. Himmelberg, Measurable relations, Fund. Math., 87 (1975), 53-72. 2, 2.12, 3

[9] H.-P. Huang, S. Radenović, Common fixed point theorems of generalized Lipschitz mappings in cone b-metric spaces over Banach algebras and applications, J. Nonlinear Sci. Appl., 8 (2015), 787-799. 1 
[10] L.-G. Huang, X. Zhang, Cone metric spaces and fixed point theorems of contractive mappings, J. Math. Anal. Appl., 332 (2007), 1468-1476. 1

[11] N. Hussain, A. Latif, N. Shafqat, Weak contractive inequalities and compatible mixed monotone random operators in ordered metric spaces, J. Inequal. Appl., 2012 (2012), 20 pages. 1

[12] N. Hussain, M. H. Shah, KKM mappings in cone b-metric spaces, Comput. Math. Appl., 62 (2011), 1677-1684. 1, 2.2, 2.3

[13] B.-H. Jiang, Z.-L. Cai, J.-Y. Chen, H.-P. Huang, Tripled random coincidence point and common fixed point results of generalized Lipschitz mappings in cone b-metric spaces over Banach algebras, J. Nonlinear Sci. Appl., 10 (2017), 465-482. 1

[14] E. Karapınar, P. Kumam, W. Sintunavarat, Coupled fixed point theorems in cone metric spaces with a c-distance and applications, Fixed Point Theory Appl., 2012 (2012), 19 pages. 1

[15] E. Karapinar, N. V. Luong, Quadruple fixed point theorems for nonlinear contractions, Comput. Math. Appl., 64 (2012), 1839-1848. 2.9

[16] E. Karapinar, N. V. Luong, N. X. Thuan, Coupled coincidence points for mixed monotone operators in partially ordered metric spaces, Arab. J. Math. (Springer), 1 (2012), 329-339. 1

[17] P. Kumam, Random common fixed points of single-valued and multivalued random operators in a uniformly convex Banach space, J. Comput. Anal. Appl., 13 (2011), 368-375.

[18] P. Kumam, S. Plubtieng, Random coincidence and random common fixed points of nonlinear multivalued random operators, Thai J. Math., 5 (2007), 155-163

[19] P. Kumam, S. Plubteing, Random common fixed point theorems for a pair of multi-valued and single-valued nonexpansive random operators in a separable Banach space, Indian J. Math., 51 (2009), 101-115.

[20] T.-C. Lin, Random approximations and random fixed point theorems for non-self-maps, Proc. Amer. Math. Soc., 103 (1988), 1129-1135. 1

[21] H. Liu, S.-Y. Xu, Cone metric spaces with Banach algebras and fixed point theorems of generalized Lipschitz mappings, Fixed Point Theory Appl., 2013 (2013), 10 pages. 1

[22] E. J. McShane, R. B. Warfield Jr., On Filippov's implicit functions lemma, Proc. Amer. Math. Soc., 18 (1967), 41-47. 3

[23] J. Merryfield, B. Rothschild, J. D. Stein Jr., An application of Ramsey's theorem to the Banach contraction principle, Proc. Amer. Math. Soc., 130 (2002), 927-933. 1

[24] Z. Mustafa, H. Aydi, E. Karapinar, Mixed g-monotone property and quadruple fixed point theorems in partially ordered metric spaces, Fixed Point Theory Appl., 2012 (2012), 19 pages. 2.10

[25] S. Phiangsungnoen, Ulam-Hyers stability and well-posedness of the fixed point problems for contractive multi-valued operator in b-metric spaces, Commun. Math. Appl., 7 (2016), 241-262. 1

[26] S. Radenović, Common fixed points under contractive conditions in cone metric spaces, Comput. Math. Appl., 58 (2009), 1273-1278.

[27] H. Rahimi, G. Soleimani Rad, P. Kumam, Coupled common fixed point theorems under weak contractions in cone metric type spaces, Thai. J. Math., 12 (2014), 1-14. 1

[28] W. Rudin, Functional analysis, Second edition, International Series in Pure and Applied Mathematics, McGrawHill, Inc., New York, (1991). 2.4, 2.5

[29] İ. Şahni, M. Telci, Fixed points of contractive mappings on complete cone metric spaces, Hacet. J. Math. Stat., 38 (2009), 59-67. 1

[30] W. Shatanawi, F. Awawdeh, Some fixed and coincidence point theorems for expansive maps in cone metric spaces, Fixed point Theory Appl., 2012 (2012), 10 pages. 1

[31] W. Shatanawi, Z. Mustafa, On coupled random fixed point results in partially ordered metric spaces, Mat. Vesnik, 64 (2012), 139-146. 1

[32] Y.-H. Shen, D. Qiu, W. Chen, Fixed point theorems in fuzzy metric spaces, Appl. Math. Lett., 25 (2012), 138-141. 1

[33] L. Shi, S.-Y. Xu, Common fixed point theorems for two weakly compatible self-mappings in cone b-metric spaces, Fixed Point Theory Appl., 2013 (2013), 11 pages.

[34] W. Sintunavarat, Y. J. Cho, P. Kumam, Common fixed point theorems for c-distance in ordered cone metric spaces, Comput. Math. Appl., 62 (2011), 1969-1978. 1

[35] W. Sintunavarat, P. Kumam, Y. J. Cho, Coupled fixed point theorems for nonlinear contractions without mixed monotone property, Fixed Point Theory Appl., 2012 (2012), 16 pages. 1

[36] A. Špaček, Zufällige Gleichungen, (German) Czechoslovak Math. J., 5 (1955), 462-466. 1

[37] T. Suzuki, A generalized Banach contraction principle that characterizes metric completeness, Proc. Amer. Math. Soc., 136 (2008), 1861-1869. 1

[38] S.-Y. Xu, S. Radenović, Fixed point theorems of generalized Lipschitz mappings on cone metric spaces over Banach algebras without assumption of normality, Fixed point Theory Appl., 2014 (2014), 12 pages. 2.6, 2.7, 2.8 